\title{
Stochastic Modeling of Unloading and Loading Operations at a Container Terminal using Automated Lifting Vehicles
}

\author{
Debjit Roy ${ }^{1}$ \\ Production and Quantitative Methods Area, \\ Indian Institute of Management Ahmedabad, \\ Gujarat 380015, India \\ René de Koster \\ Department of Technology and Operations Management, \\ Rotterdam School of Management, Erasmus University, \\ Rotterdam 3062PA, The Netherlands
}

\begin{abstract}
With growing worldwide trade, container terminals have grown in number and size. Many new terminals are now automated to increase operational efficiency. The key focus is on improving seaside processes, where a distinction can be made between single quay crane operations (all quay cranes are either loading or unloading containers) and overlapping quay crane operations (some quay cranes are loading while others are unloading containers). From existing studies, it is not clear if the design insights obtained from analyzing single operations, such as optimal stack layout, are consistent with the insights obtained from analyzing overlapping operations. In this paper, we develop new integrated stochastic

\footnotetext{
${ }^{1}$ Corresponding Author, Email: debjit@iimahd.ernet.in, Phone: +91-7966324823, Fax: +91-7966326896
} 
models for analyzing the performance of overlapping loading and unloading operations that capture the complex stochastic interactions among quayside, vehicle, and stackside processes. Using these integrated models, we are able to show that that there are stack layout configurations that are robust for both single (either loading or unloading) and for overlapping (both loading and unloading) operations.

\section{Keywords}

Logistics, Queuing, Transportation, Uncertainty modelling

\section{Introduction and Literature Review}

With over $90 \%$ of the global trade carried over sea, the maritime containerization market is projected to reach 731 million TEU by 2017 (Global Industry Analysts Inc. [2013]). Sea transport is regarded as the most economical mode of transport especially for the movement of international cargo. As shipping liners are demanding higher service levels, container terminals need to be designed to optimize performance objectives, such as, for example, reducing service times and improving crane productivity. Some recent technological advancements include multi-trolley quay cranes that are developed to improve the crane productivity, multiple automated stacking cranes per stack block to improve efficiencies at the stackside, and automated lifting vehicles to improve container transport times. While technology plays a big role in improving system performance, optimal design principles help to leverage the potential of an automated technology to the fullest.

In this study, we assume that the handling of containers within the terminal is executed by a fleet of quay cranes (QCs), automated lifting vehicles (ALVs), and automated stacking cranes (ASCs)(See Figure 1). When a ship berths, one or multiple quay cranes are assigned to the ship to unload the containers depending on the size of the ship. The quay cranes 
position the containers at the quayside buffer locations. ALVs transfer the containers from the quayside to a prespecified stack block. After reaching the stack block buffer lanes, the inbound containers are dropped off. Next, an ASC picks up the container and stacks it in the stack block. Due to varying bay sizes in the vessel (greater at the middle compared to the front and the rear), the container unloading operation is typically completed sooner in the front and the rear areas of the ship than in the other areas. The quay cranes that previously unloaded inbound containers from the front and rear portions of the ship are assigned to load the outbound containers in the empty locations of the ship. This assignment marks the inception of the overlapping phase, when both loading and unloading operations are executed simultaneously. When all unloading has finished, the overlapping phase transfers to the loading phase, when all cranes load the vessel. Cranes that have finished their bays can be transferred to adjacent vessels.

Managing simultaneous loading and unloading of containers is a complex task because this operation demands coordination among the ALVs, the QCs, and the ASCs that transfer the containers from the stackside to the quayside or vice versa. The terminal throughput performance with overlapping operations depends on multiple design and operational control parameters. For instance, the vehicle to container assignment depends on the vehicle's current location and the next task to be performed. A vehicle that has just completed unloading operation and dwells near the stack lanes may be preferred if the next operation is a loading operation. Further, throughput performance may be affected by the topology of the vehicle travel path (including the number of shortcuts between the quayside and the stackside) and the configuration of the stack block layout. The sequence in which quay cranes are assigned to process loading and unloading operations may also impact throughput times, due to the topology of the vehicle guide path.

Many researchers have focused on performance analysis of specific container terminal 


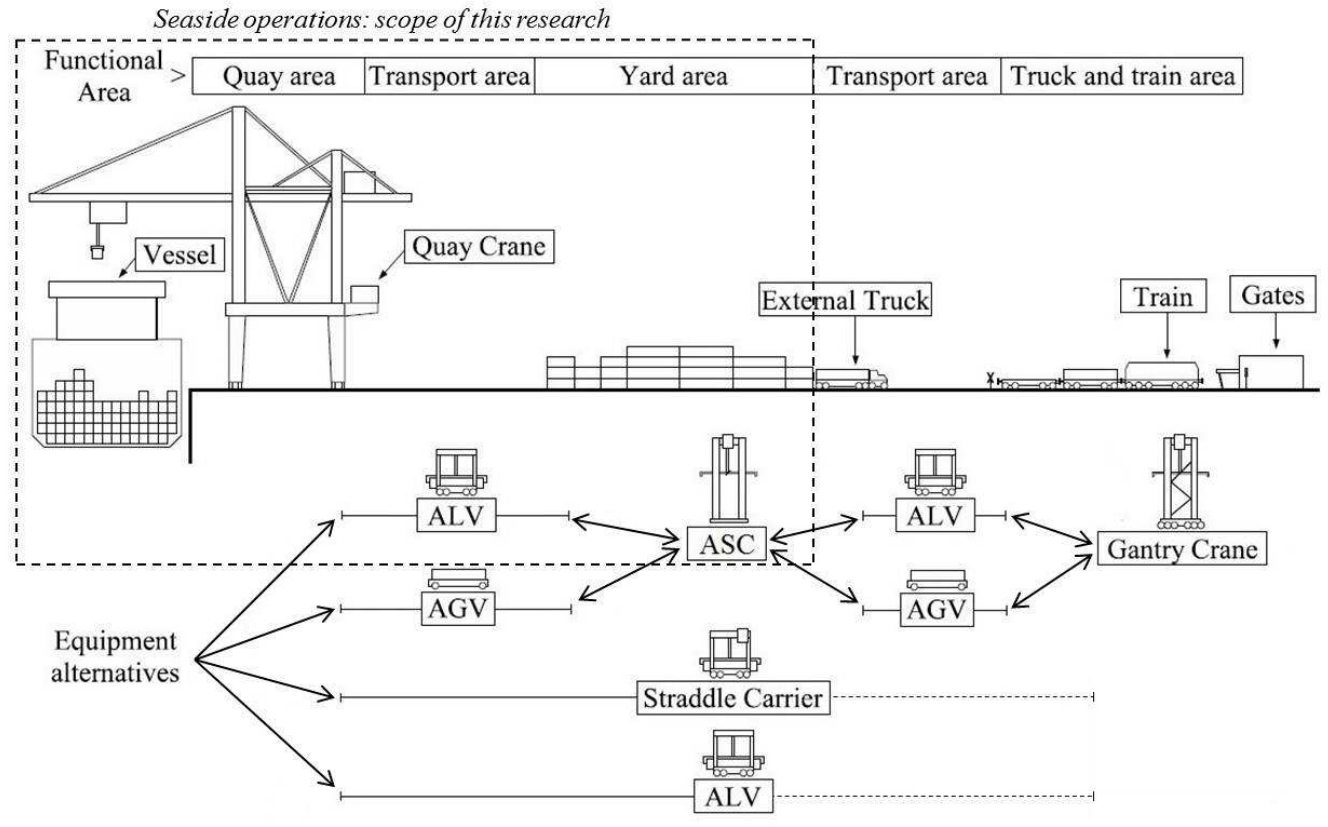

Figure 1: Functional areas and resources at a container terminal, Brinkmann [2010] and Meisel [2009]

design aspects. For an overview of the literature on container terminal modeling, see Gharehgozli et al. [2014b]; Stahlbock and Voß [2008]; Steenken et al. [2005]; Vis and De Koster [2003]. However, little research has focused on modeling and developing design insights for container terminal under joint loading and unloading operations. Existing research on modeling and design of container terminal operations can be broadly classified into two categories depending on the type of system under investigation: 1) isolated system research, focusing on one of the three processes: quayside, vehicle transport, or stackside operations, and 2) integrated system research, where the three processes and their interactions are studied together as one integrated operation. We discuss both streams of research.

Research on isolated systems focuses on developing optimization and simulation models 
to address operational issues such as scheduling of container storage and retrieval operations (Vis and Roodbergen [2009], Gharehgozli et al. [2014a]), routing algorithms for transfer cranes (Kim and Kim [1999]), quay crane scheduling (Kim and Park [2004], Liang et al. [2009]), and workload management at yard cranes ( $\mathrm{Ng}$ [2005]).

The second stream of research focuses on evaluating design decisions of systems in interaction. Using detailed simulation models, researchers have studied the performance and cost trade-offs using different type of vehicles for intra-terminal container transport: multi-trailers, automated guided vehicles (AGVs) or ALVs (see Duinkerken et al. [2007], Vis and Harika [2004]). Simulation models developed to analyze operational rules such as the effect of vehicle dispatching policies, e.g., by De Koster et al. [2004]. Briskorn et al. [2007] show that an inventory-based vehicle dispatching policy is more robust than a due-date based vehicle dispatching policy. Bae et al. [2011] compare the operational performance of an integrated system with two types of vehicles (ALVs and AGVs). Through simulation experiments, they show that the ALVs reach the same productivity level as the AGVs using fewer vehicles due to the self-lifting capability. Canonaco et al. [2008] simulate the assignment of QCs to the holds of a vessel, the assignment of straddle carriers to the QCs, and the sequencing of loading and unloading operations. Detailed simulation models have also been developed to analyze strategies for controlling dual-load vehicles and yard crane systems in real time within a fully-integrated, stochastic container terminal environment (see Petering et al. [2009], Petering [2010]), and also understanding the effect of yard allocation policies on QC throughput rate (see Petering [2009]).

Some researchers have focused on an analytical approach. Li and Vairaktarakis [2004] develop algorithms to optimize the time needed for simultaneous loading and unloading operations with a fleet of trucks that move the containers from the quayside (single QC) to the stackside and vice versa. Goodchild and Daganzo [2006] attempt to reduce the 
turn-around time of a ship by formulating the double-cycling problem (QC loading and unloading containers in one cycle) as a scheduling problem, which can be solved optimally. Bish [2003] examines the vehicle dispatching problem for loading and unloading containers to and from ships and develops greedy heuristic algorithms to determine job schedules, which are optimal for a single ship-single crane combination, and minimizes the overall dwell time of a ship at the terminal. Analytical models have also been developed to analyze terminal design decisions. For instance, Hoshino et al. [2007] proposed an optimal design methodology for an AGV transportation system by using a combination of closed queuing networks.

To date, practitioners have primarily developed detailed simulation models to design new terminals or improve the efficiency of existing terminal operations (see TBA BV [2010]). However, while simulations can help for detailed analysis, the complexity and interactions involved make such models prohibitively expensive and time consuming if used for generating and selecting designs (Edmond and Maggs [2009]). In this regard, integrated queuing network models can help to rapidly evaluate and identify optimal container terminal designs (with a short model development and setup time). Roy and De Koster [2012] developed an analytical, integrated model of a container terminal, focusing on either loading or unloading operations. Using an analytical model, they identified the optimal choice for the vehicle dwell point, the optimal layout of the stack, and the vehicle guide path. However, in the case of many deep-sea vessels, substantial time is spent in the overlapping phase, when some QCs may still be unloading the vessel while others have already started loading certain bays of the vessel. It is important to start loading the vessel as early as possible to minimize berthing time. The contribution of this research is to develop an integrated analytical model (using a combination of open and semi-open queuing networks) for analyzing the overlapping unloading and loading operations in a container terminal and to develop 
an efficient (parametric decomposition-based) high-quality solution approach.

It is not at all clear whether design decisions for single operations (such as the design of the stack layout) also hold true for overlapping operations. As an example, we evaluate one such design decision in this paper: what is the most efficient stack configuration (number of stack blocks, number of rows per stack block, number of bays, and number of tiers per block) for overlapping operations.

The rest of this paper is organized as follows. In Section 2, we describe the overlapping operations at a container terminal. The queuing network model developed to analyze the performance of a terminal with integrated overlapping operations is described in Section 3. In Sections 4, 5, and 6, we develop models for analyzing the $\mathrm{QC}$, vehicle movement, and ASC processes in isolation. The model integration is described in Section 7. The numerical experiments and design insights are presented in Sections 8 and 9, respectively. The conclusions are drawn in Section 10.

\section{Description of Overlapping Operations}

In Figure 2, we describe the overlapping operations at the quayside. Consider a scenario with three quay cranes $\left(Q C_{1}, Q C_{2}\right.$, and $\left.Q C_{3}\right)$. The x-axis denotes time. The three $\mathrm{QCs}$, $Q C_{1}, Q C_{2}$, and $Q C_{3}$, start unloading containers from the vessel at time zero. Note that $Q C_{1}$ completes the unloading operation and starts to load containers at time $t_{1}$ while $Q C_{2}$ and $Q C_{3}$ are still unloading containers. Likewise, at time $t_{4}, Q C_{1}$ completes its ship loading operation and is assigned to another ship $S_{2}$ for unloading, while $Q C_{2}$ and $Q C_{3}$ are still loading containers into $S_{1}$.

Based on Figure 2, Table 1 includes the operational phases for the three quay cranes 


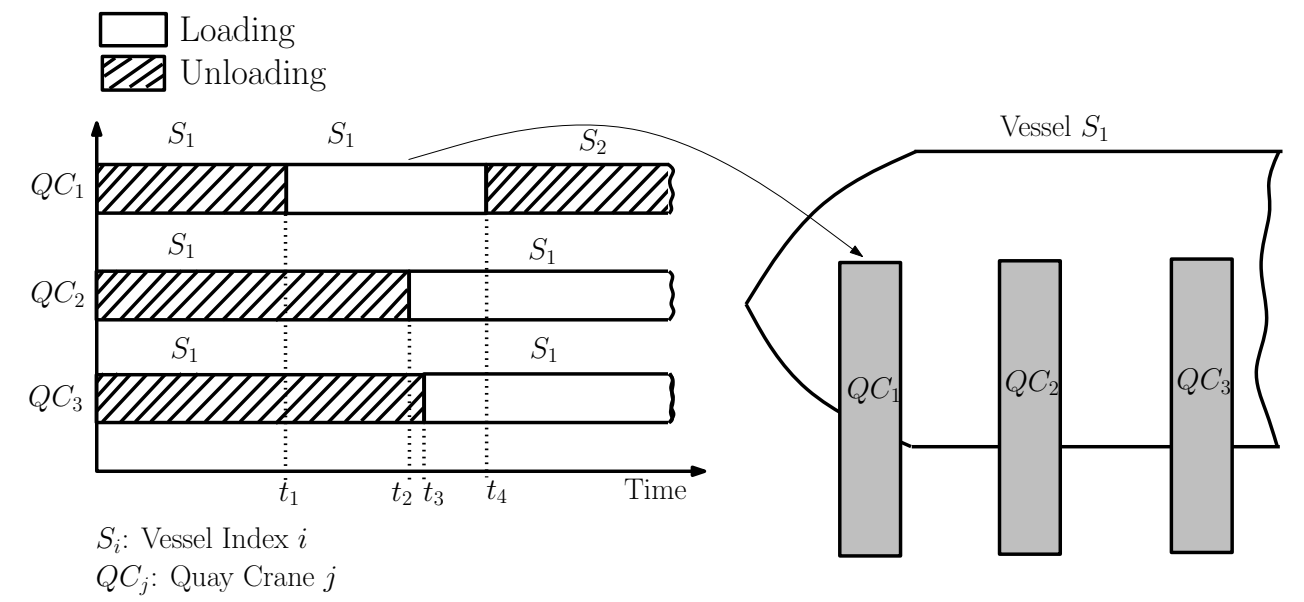

Figure 2: Illustration of overlapping operations at quayside

at various time intervals. For instance, the time intervals: $\left[0, t_{1}\right),\left[t_{1}, t_{2}\right)$, and $\left[t_{2}, t_{3}\right)$ correspond to phase 1,2 , and 3 , respectively. In this research, we develop an analytical model for a terminal configuration corresponding to a particular operational phase (time interval). For each operational phase, some QCs are dedicated to unloading containers from a vessel whereas the others load containers onto a vessel. Therefore, to determine aggregated performance measures (expected throughput time of unloading or loading a container), these measures should be weighted across the different time intervals. Also note that we model the berthing space of one vessel. Once loading/unloading operations on a vessel are complete, another vessel arrives and loading/unloading operations are repeated again.

Table 1: Phases of overlapping operations for vessel, $S_{1}$

\begin{tabular}{clllll}
\hline QC/Time & {$\left[0, t_{1}\right)$} & {$\left[t_{1}, t_{2}\right)$} & {$\left[t_{2}, t_{3}\right)$} & {$\left[t_{3}, t_{4}\right)$} & {$\left[t_{4}, t_{5}\right)$} \\
\hline$Q C_{1}$ & Unload & Load & Load & Load & - \\
$Q C_{2}$ & Unload & Unload & Load & Load & Load \\
$Q C_{3}$ & Unload & Unload & Unload & Load & Load \\
\hline
\end{tabular}

While the basic nodes of the queuing model remain the same across different time periods, 
the routing of the containers in the network varies depending on the operational phase. Since the performance of $\mathrm{QC}$ operation depends on the interaction between the transport and storage systems, we need an integrated model to capture these stochastic interactions. The steady-state performance measures such as the expected container handling times can be used to measure internal efficiencies, benchmark terminal performance, and develop design insights for overlapping operations.

In the next section, we describe the modeling assumptions and the model development approach for the quayside, vehicle transport, and stackside processes.

\section{Queuing Network Model: Assumptions and Approach}

\subsection{Assumptions}

To analyze the performance of a container terminal with overlapping operations, we consider an ALV-based terminal whose layout is based on input data from the ECT terminal in Rotterdam, (see De Koster et al. [2004]). We consider the space required for berthing a jumbo vessel. However, the layout can be easily extended to consider the berthing of multiple ships. The layout shown in Figure 3 broadly comprises three areas: quayside, vehicle transport, and stackside. The quayside area includes $N_{q}$ quay cranes that are spaced equidistant from each other. The vehicle transport area includes a guided path that supports the movement of the ALVs (see Table 2 for notations used in the layout). The unidirectional travel path joins the quayside buffer lanes and the stackside buffer lanes. The travel path has a set of shortcuts that reduces travel time from quayside to stackside. The shortcut paths run from quayside to stackside, but not in the opposite direction. The existing literature, for example, Vis and Harika [2004], typically considers a travel path 
with a single loop, which undoubtedly simplifies the model, but reduces its validity. Our layout captures the topological constraints imposed at modern container terminals.

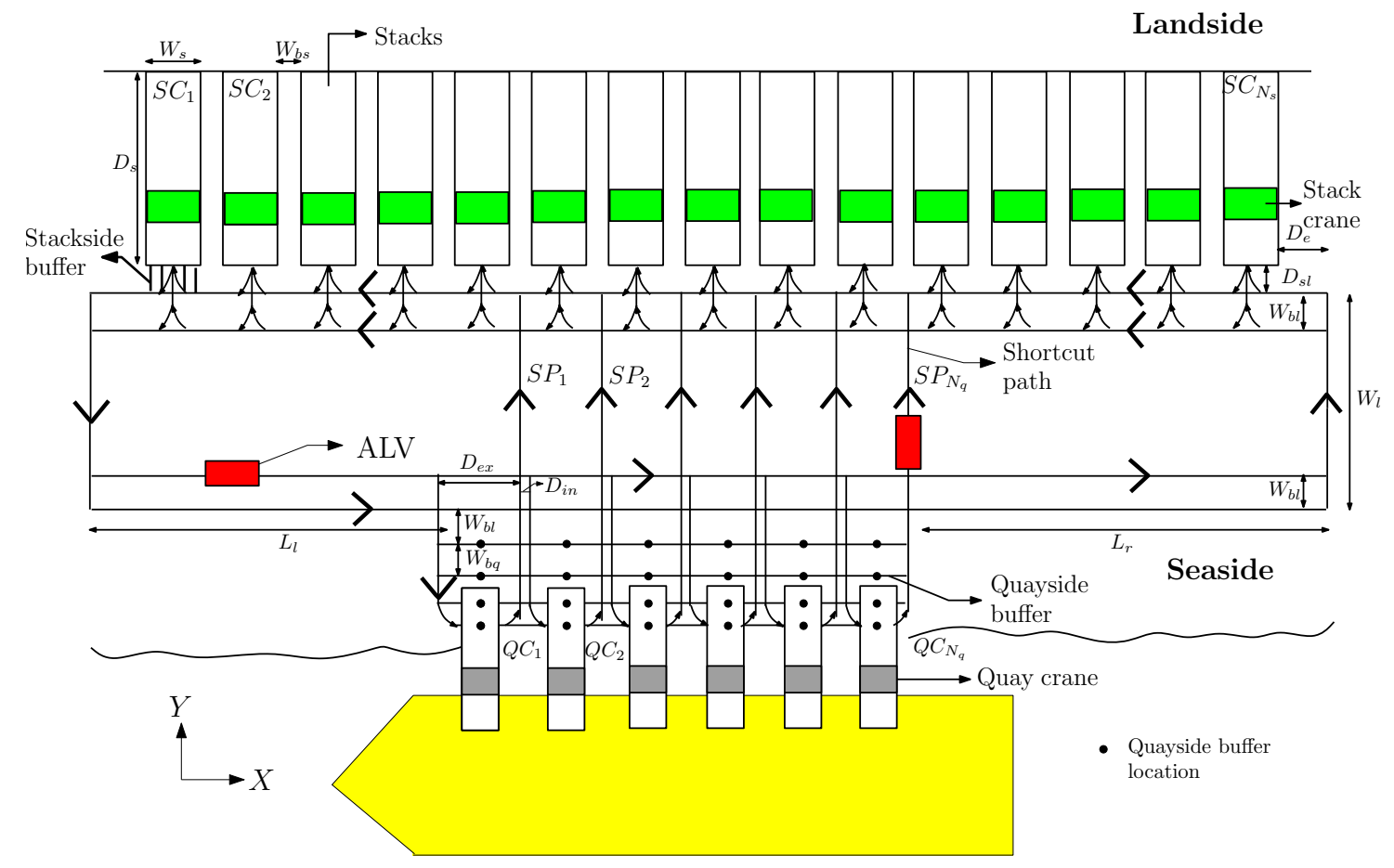

Figure 3: Layout of the container terminal used in this research

During unloading operations, a container typically goes through six handling phases: (1) it waits on the vessel for the QC to handle it, (2) it is unloaded by the QC, (3) it waits for an ALV in the QC buffer lane, (4) it is transported by the ALV, (5) it waits on the ground for ASC service, and (6) it is loaded by an ASC and put into a stack. The loading operations are executed in reverse order, where the container first waits on the stack for the ASC and the loading process concludes when the QC loads the container on the vessel. The load and unload throughput times includes the time spent by the container during the six phases. We now state the modeling assumptions for the three processes present in the model for overlapping operations. 
Table 2: Notations used in Figure 3 as well as used to obtain the service time expressions for the vehicle transport

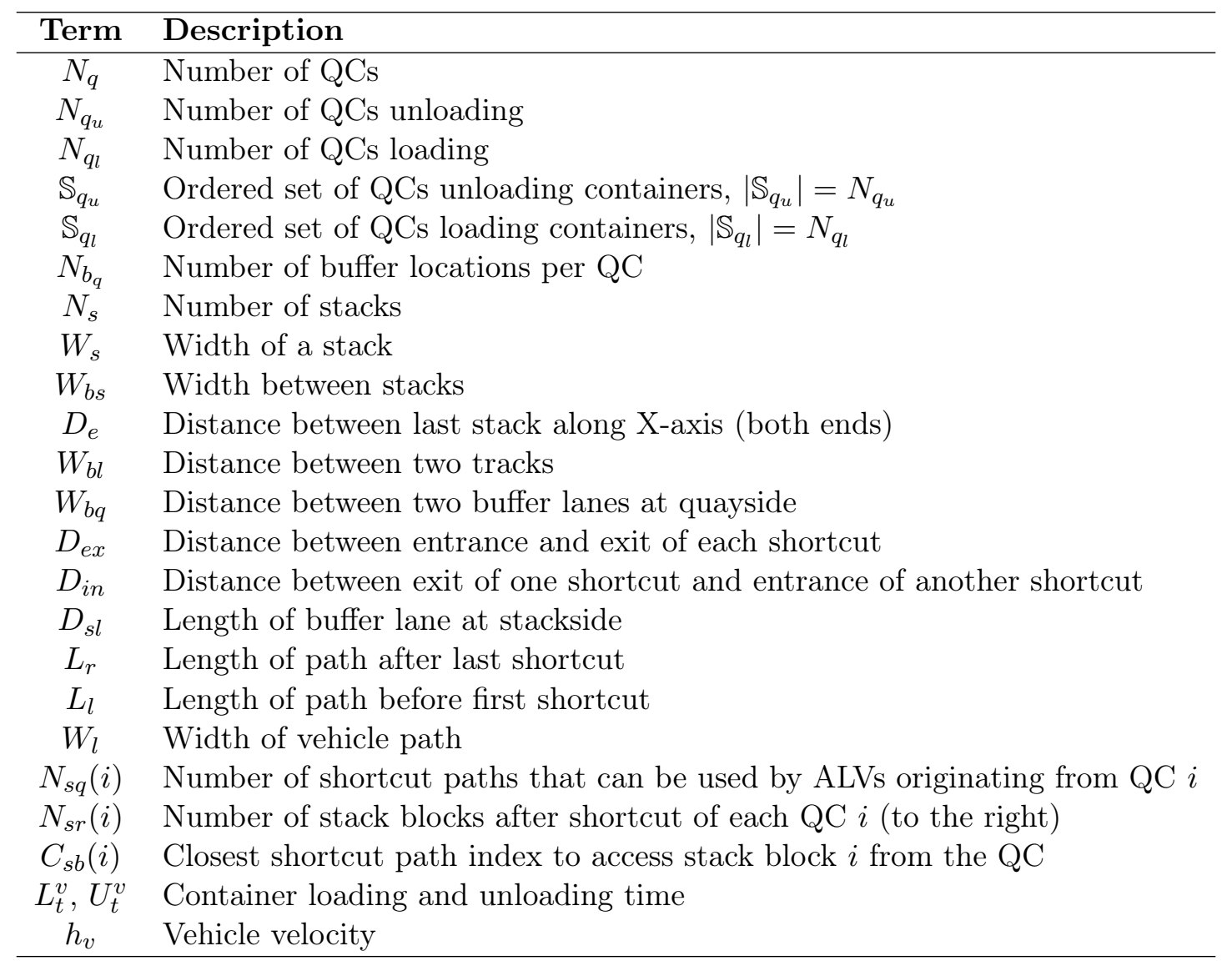

Quayside Process: We consider QCs with only one trolley. Each QC processes either unloading or loading transactions during the phase of an overlapping operation (single cycle). The container arrival processes for both loading (stackside) and unloading (quayside) transactions are modeled as bursty $(\mathrm{CV}>1$ or $\mathrm{CV}=1$, Poisson).

The containers to be unloaded are randomly assigned to any QC allocated to process unloading operations. Likewise, the containers to be loaded are randomly assigned to any QC allocated to process loading operations. The dwell point of the QC trolley is the point 
of service completion. We consider infinite buffer lane space for vehicles parking near the quay cranes. Note that this assumption is not unreasonable because vehicles can park near the quay cranes if there is no availability in the buffer space.

Vehicle Transport Process: We only consider ALVs, which can autonomously pick up and set down containers in the absence of other equipment. Vehicles have a carrying capacity of one container (TEU). Vehicles are not dedicated to QCs; they are allowed to process loading and unloading operations consecutively (double cycling). Note that a vehicle at the stackside may be better suited to process a loading operation than an unloading operation. Therefore, we also consider preferential vehicle assignment later. Vehicles follow a pointof-service-completion dwell point policy i.e., they dwell at the stackside after processing unloading requests and dwell at the quayside after processing loading requests.

Stackside Process: There is one ASC per stack block, which can process both loading and unloading operations in a single phase of the overlapping operations. Note that this assumption holds in practice, because a container may need to be unloaded from a stack and another container may need to be loaded into the same stack. Containers are randomly assigned to any ASC during loading and unloading operations so that the workload is uniformly distributed among ASCs. The total number of storage locations is fixed, but we vary the number of stacks $\left(N_{s}\right)$, number of rows per stack $\left(N_{r}\right)$, bays per stack $\left(N_{b}\right)$, and tiers per stack $\left(N_{t}\right)$. To capture the variation in service time of ASC operations (hoisting, lowering, trolleying, and gantrying) involved in placing containers in the stack, we generate a random storage location (stack, row, bay, and tier) for each unloaded and loaded container. The dwell point of the ASCs is the point of service completion. We again assume infinite buffer space for vehicle parking at each ASC location. 


\subsection{Modeling Approach}

We now briefly describe the approach to develop the integrated analytical model for a container terminal with overlapping operations. We first develop the individual models the QCs, the ASCs, and the ALV movement. The QC and the ASC resources are modeled using single server stations. However, modeling the ALV movement is challenging. The ALVs receive processing requests from QC resources for unloading and from ASC resources for loading. We model interactions between ALVs and container arrivals using a multi-class semi-open Queuing Network with class-switching. The interactions among the three resource types (QCs, ASCs, and ALVs) are captured using the first and the second moment information for the inter-departure times from the resources. Then we link the three submodels using an iterative convergence (IC) algorithm to capture the stochastic interactions among the upstream and downstream processes. We now introduce three high-level steps (see Figure 4). In Step 1, we analyze the performance of the QCs unloading containers

from the vessel. In Step 2, we use the departure process information from the QCs to analyze the performance of the vehicle subnetwork and the ASC resources processing both loading and unloading operations. Finally, we use the departure process information for the load (export) containers from the vehicle subnetwork to analyze the performance of QCs loading containers into the vessel. The steps of the modeling approach can be summarized as follows:

Step 1: Evaluate the performance of the QCs processing unloading operations

Step 2: Replace the vehicle closed queuing subnetwork with an equivalent load dependent queue and evaluate the performance of the vehicle network and stack cranes through an iterative convergence algorithm

- Estimate the arrival process and departure process for the vehicle network 
- Evaluate the performance of the vehicle network using a CTMC with a Cox-2 inputs

- Evaluate the performance of the ASCs

Step 3: Evaluate the performance of the QCs processing loading operations

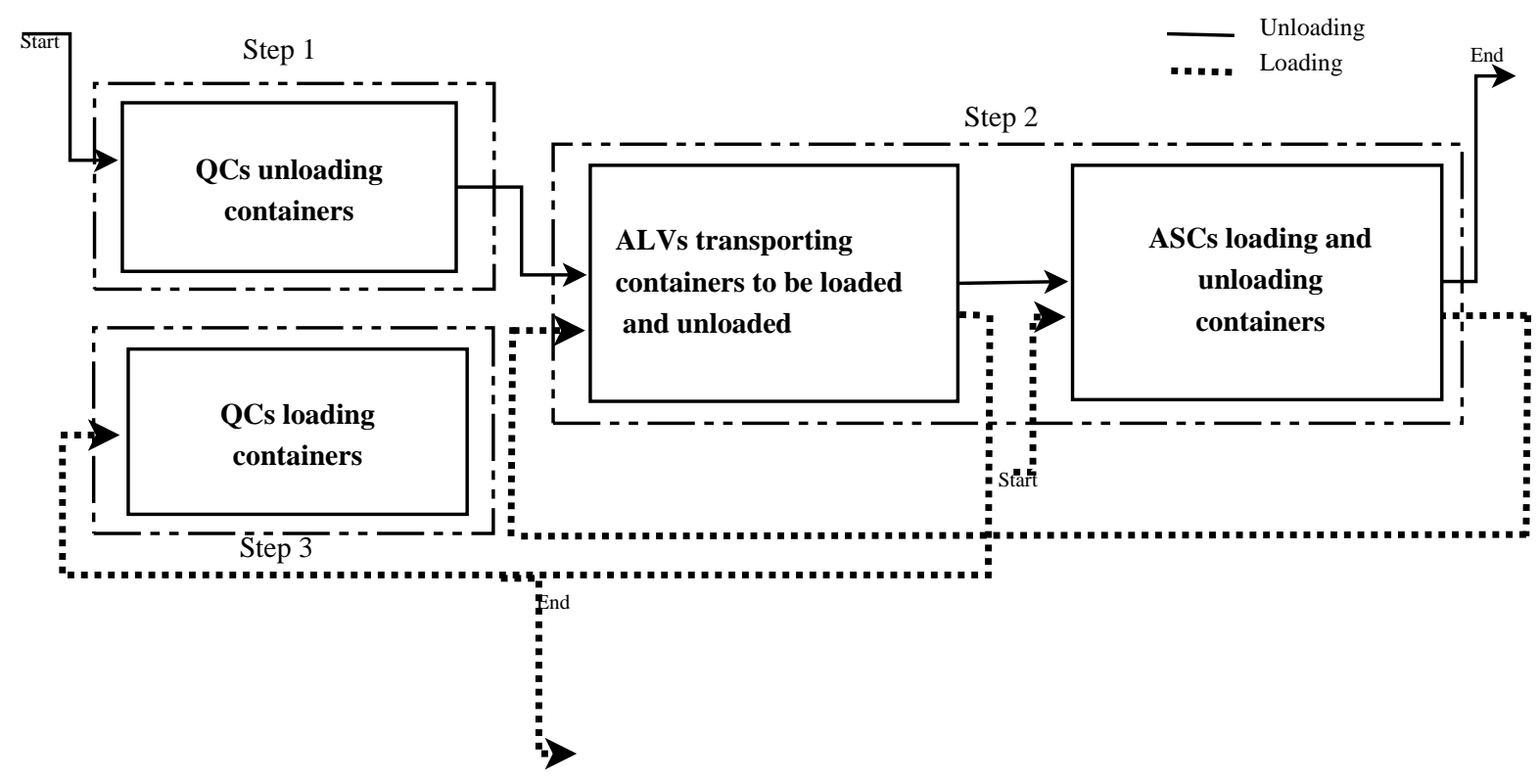

Figure 4: Stochastic interactions among the resources and the modeling approach

We describe the models for the individual processes in the subsequent sections. In the model description, we commonly use terms of the form, $p_{i_{j_{k}}}(m)$ (see Table 3), where $p$ could take one of the following forms: $\lambda^{-1}$ (container mean inter-arrival or inter-departure times depending on the value of component $i$ ), $c^{2}$ (squared coefficient of variation of inter-arrival or inter-departure times depending on the value of component $i$ ), $\mu^{-1}$ (mean service time for the QC or ASC depending on component $j$ ), $\lambda$, and $\mu$ (arrival and service rates of the containers to the QC, ALV, or ASC depending on component $j$ ). Symbol $i$ represents either $a$ (arrival) or $d$ (departure). Alternatively, symbol $i$ is omitted if the process itself is consid- 
ered - hence not the arrivals to, or the departures from the process. Symbol $j$ corresponds to one of the three resources $q(\mathrm{QC}), s$ (ASC), or $t$ (ALV). Symbol $k$ corresponds to either $u$ unloading or $l$ loading operation. Finally, symbol $m$ corresponds to the resource index.

Table 3: Description of frequently used term of the form $p_{i_{j_{k}}}(m)$. For instance, $c_{a_{q_{u}}}^{2}(i)$ denotes the squared coefficient of variation of the inter-arrival $(a)$ times for the QC $(q)$ index $i$ processing unloading $(u)$ operations.

\begin{tabular}{cl}
\hline Symbol & Values \\
\hline$p$ & $\lambda^{-1} / c^{2} / \mu^{-1} / \lambda / \mu$ \\
$i$ & $a / d($ arrival/departure) \\
$j$ & $q / s / t(\mathrm{QC} / \mathrm{ASC} /$ transport (ALV)) \\
$k$ & $u / l($ unloading/loading) \\
$m$ & resource index \\
\hline
\end{tabular}

\section{Model for QCs}

One set of QCs are dedicated to unloading containers from the vessel while the rest are dedicated to loading containers onto the vessel. To unload a container, the QC's trolley moves from its dwell point to the container pick-up location in the vessel, and repositions the container at the quayside buffer lane. Likewise, to load a container, the QC's trolley moves from its dwell point to the container pick-up location at the buffer lane, and then positions the container in the vessel. The queueing model for the QC models the congestion at the quayside. Both type of QC resources, loading and unloading, are modeled as $G I / G / 1$ queues.

The objective of the QC queuing model (for unloading operations) is to estimate the performance measures, and the squared coefficient of variation (SCV) of the inter-departure times from the $\mathrm{QC}$ resources $\left(c_{d_{q_{u}}}^{2}(i)\right)$. The inputs to the model are the first moment and 
the SCV of the inter-arrival times to the QCs $\left(\lambda_{a_{q_{u}}}^{-1}(i)\right.$ and $\left.c_{a_{q_{u}}}^{2}(i)\right)$, and the $\mathrm{QC} i$ service times $\left(\mu_{q_{u}}^{-1}(i)\right.$ and $c_{s_{q_{u}}}^{2}(i)$ where $\left.i=\left\{1, \ldots, N_{q_{u}}\right\}\right)$. Let $\lambda_{a}^{u}$ be the overall container arrival rate for unloading operations. Due to the thinning process, the arrival process to the QCs assigned for unloading operations are also Poisson with rate $\lambda_{a_{q_{u}}}(i)=\frac{\lambda_{a}^{u}}{N_{q_{u}}}$. With these input parameters, each $\mathrm{QC}$ queue is modeled as a $G I / G / 1$ queue and the performance measures such as expected waiting time $\left(\mathbb{W}_{q_{u}}(i)\right)$, crane utilization $\left(\mathbb{U}_{q_{u}}(i)\right)$ and the SCV of the inter-departure times are estimated using two-moment approximation results, Whitt [1983] (Equations 4-5). Expected unload throughput time, $\mathbb{E}\left[T_{q}^{u}\right]$, is obtained using the sum of the expected waiting time, $\mathbb{W}_{q_{u}}(i)$, and expected service time, $\mu_{q_{u}}^{-1}(i)$. Equations 6 and 7 provide the aggregated SCV and the mean of the inter-arrival times, $c_{a_{t_{u}}}^{2}$ and $\lambda_{a_{t_{u}}}^{-1}$, for the unloaded containers to the vehicle network, respectively.

$$
\begin{aligned}
\lambda_{a_{q_{u}}}(i) & =\frac{\lambda_{a}^{u}}{N_{q_{u}}} \\
\mathbb{U}_{q_{u}}(i) & =\frac{\lambda_{a_{q_{u}}}(i)}{\mu_{q_{u}}(i)} \\
c_{a_{q_{u}}}^{2}(i) & =\frac{c_{a_{q_{u}}}^{2}}{N_{q_{u}}}+\left(1-\frac{1}{N_{q_{u}}}\right) \\
\mathbb{W}_{q_{u}}(i) & =\left(\frac{\mu_{q_{u}}^{-1}(i) \mathbb{U}_{q_{u}}(i)}{1-\mathbb{U}_{q_{u}}(i)}\right)\left(\frac{c_{a_{q_{u}}}^{2}(i)+c_{s_{q_{u}}}^{2}(i)}{2}\right) \\
c_{d_{q_{u}}}^{2}(i) & =\mathbb{U}_{q_{u}}^{2}(i) c_{s_{q_{u}}}^{2}(i)+\left(1-\mathbb{U}_{q_{u}}^{2}(i)\right) c_{a_{q_{u}}}^{2}(i) \\
c_{a_{t_{u}}}^{2} & =\sum_{i=1}^{N_{q_{u}}} \frac{\lambda_{a_{q_{u}}}(i) c_{d_{q_{u}}}^{2}(i)}{\lambda_{a}^{u}} \\
\lambda_{a_{t_{u}}}^{-1} & =\lambda_{a_{u}}^{-1}
\end{aligned}
$$

where $i \in \mathbb{S}_{q_{u}}$, the set of QCs unloading containers.

Note that the QCs loading containers onto the vessel are also modeled using $G I / G / 1$ queues. During the loading process, the containers are transferred to the QCs by the ALVs. 
The arrival process inputs to the QCs $\left(\lambda_{q_{l}}^{-1}(i)\right.$ and $\left.c_{a_{q_{l}}}^{2}(i)\right)$ are obtained from the departure process outputs from the ALV network described in Section 5. The expressions to estimate the performance measures are similar to the ones included in Equation 4 and 5.

\section{Model for Vehicle Transport}

In this section, we first describe the queuing network for the vehicle transport process, and then explain the service time expressions at the travel nodes. Finally, we describe the methodology for reducing the vehicle network to evaluate the performance measures and link the network with the QC and the ASC models.

\subsection{Description of Vehicle Transport Queuing Network}

The queuing model for the vehicle transport is described in Figure 5a. As mentioned earlier, the vehicles perform both loading $(l)$ and unloading $(u)$ operations. The arrival process input to the vehicle transport network is characterized by the first moment and the SCV of the inter-arrival times for unloading $\left(\lambda_{a_{t_{u}}}^{-1}, c_{a_{t_{u}}}^{2}\right)$ and loading operations $\left(\lambda_{a_{t_{l}}}^{-1}, c_{a_{t_{l}}}^{2}\right)$. There are two classes of vehicles depending on the vehicle start point (as in Figure A.1, Appendix A) of processing a transaction: quayside or stackside, that are denoted by index $q$ and $s$, respectively. Containers that either need to be loaded or unloaded, queue at a virtual buffer $B_{1}$ and idle vehicles queue at buffer $B_{2}$.

Depending on the type of vehicle class $(q$ or $s$ ) and the type of request processed by the vehicle $(l$ or $u)$, there are four service types. The expected service times for the four service types $q l, q u, s l$, and $s u$, are denoted by $\tau_{q l}^{-1}, \tau_{q u}^{-1}, \tau_{s l}^{-1}$, and $\tau_{s u}^{-1}$, respectively. The travel paths for the four service types are illustrated in Figure A.1. Table 4 describes the 


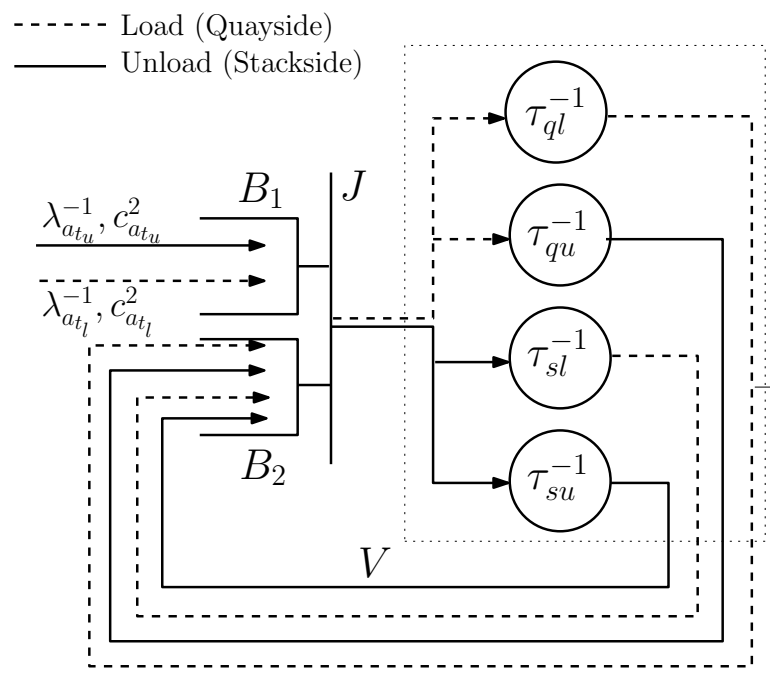

(a)
$\Pi$

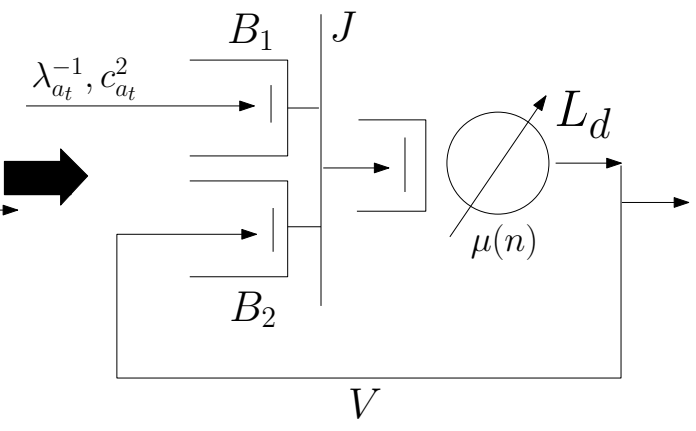

(b)

Figure 5: (a) Semi-open queuing network model for overlapping vehicle travel operations, (b) Network reduction using a load-dependent server, $L_{d}$

vehicle class switching rule for the two vehicle classes and four service types. Since vehicles are assigned randomly to the next transaction on arrival, the probability that a $q$ or an $s$ class vehicle is assigned to transport a container from quayside to stackside (unloading a container) is given by $\frac{\lambda_{a_{t_{u}}}}{\lambda_{a_{t_{u}}}+\lambda_{a_{t_{l}}}}$. Likewise, the probability that a $q$ or a $s$ class vehicle is assigned to transport a container from stackside to quayside (loading a container) is given by $\frac{\lambda_{a_{t_{l}}}}{\lambda_{a_{t_{u}}}+\lambda_{a_{t_{l}}}}$.

Table 4: Description of the vehicle class switching rule

\begin{tabular}{cccc}
\hline $\begin{array}{c}\text { Vehicle Class } \\
\text { prior to Service }\end{array}$ & $\begin{array}{c}\text { Transaction } \\
\text { Type }\end{array}$ & $\begin{array}{c}\text { Service } \\
\text { Type }\end{array}$ & $\begin{array}{c}\text { Vehicle Class } \\
\text { after Service }\end{array}$ \\
\hline Quayside $(q)$ & Load $(l)$ & $q l$ & Quayside $(q)$ \\
Quayside $(q)$ & Unload $(u)$ & $q u$ & Stackside $(s)$ \\
Stackside $(s)$ & Load $(l)$ & $s l$ & Quayside $(q)$ \\
Stackside $(s)$ & Unload $(u)$ & $s u$ & Stackside $(s)$ \\
\hline
\end{tabular}

We now describe the derivations and the expressions for the service times in the four 
vehicle service types, $q l, q u, s l$, and $s u$. Note that the expressions are developed for the container terminal topology described in Figure 3. Also observe that $\mathbb{S}_{q_{u}}$ and $\mathbb{S}_{q_{l}}$ are ordered sets (see Table 2); for instance if $\mathbb{S}_{q_{u}}=\{2,3,4\}$, we denote the value of the $i$-th element by $\mathbb{S}_{q_{u}}(i)\left(\mathbb{S}_{q_{u}}(1)=2, \mathbb{S}_{q_{u}}(2)=3, \mathbb{S}_{q_{u}}(3)=4\right)$. Further, $N_{s q}(i)$ denotes the number of shortcut paths that can be used by ALVs originating from QC $i$, respecting the single direction travel on the path. Therefore, from Figure $3, N_{s q}(1)=6$. Likewise, $N_{s r}(i)$ denotes the number of stack blocks to the right of each QC $i$. In Figure 3, $N_{s r}(1)=10$. The expected service times for the four service types $q l, q u, s l$, and $s u$ are obtained in Appendix A.

Once we obtain the expected service times at the four service nodes, we can evaluate the performance of the vehicle network in isolation. However, to link the vehicle network model with the upstream QC resources and the downstream ASC resources, we also need the higher moments of the inter-departure times from the vehicle network. This motivates the need to reduce the vehicle network to a load-dependent station, which is the subject of the next subsection. Estimating the higher moments from the original vehicle queue network is very complex. Hence, we adopt a two-step approximation procedure to estimate the second moment from the vehicle queue network. In the first step, we reduce the subnetwork from the vehicle queue network (represented by $\Pi$ in Figure 5a) to an equivalent load-dependent station, $L_{d}$. Note that in the original network, the subnetwork $\Pi$ is product-form due to the presence of Infinite Server nodes with service time distributions having rational Laplace transforms (Chandy et al. [1975],Baskett et al. [1975]). Therefore, the subnetwork satisfies the conditions stated in the BCMP Theorem. We adopt the embedded Markov chain analysis procedure developed by Roy [2011] to estimate the second moment of interdeparture times from a semi-open network containing one load-dependent station. The performance measures such as vehicle utilization $\left(\mathbb{U}_{t}\right)$, and expected throughput times for loading and unloading containers $\left(\mathbb{T}_{t}^{l}\right.$ and $\left.\mathbb{T}_{t}^{u}\right)$, are given in Equations 8-10. We define a 
term $\gamma$ as the ratio between the busy vehicles processing unloading requests versus the busy vehicles processing loading requests $\left(\gamma=\frac{\hat{Q}_{q u}+\hat{Q}_{s u}}{\hat{Q}_{q l}+\hat{Q}_{s l}}\right)$. We use $\gamma$ to approximate the expected number of busy vehicles processing unloading and loading requests $\left(Q_{u}\right.$ and $\left.Q_{l}\right)$ in the semi-open queuing network.

$$
\begin{aligned}
\mathbb{U}_{t} & =\frac{Q_{b}}{V} \\
\mathbb{E}\left[T_{t}^{u}\right] & =\frac{Q_{u}}{\lambda_{a_{t_{u}}}}+\frac{\mathbb{L}_{t}}{\lambda_{a_{t_{u}}}+\lambda_{a_{t_{l}}}} \\
\mathbb{E}\left[T_{t}^{l}\right] & =\frac{Q_{l}}{\lambda_{a_{t_{l}}}}+\frac{\mathbb{L}_{t}}{\lambda_{a_{t_{u}}}+\lambda_{a_{t_{l}}}}
\end{aligned}
$$

where $Q_{u} \sim \frac{\gamma Q_{b}}{1+\gamma}$ and $Q_{l} \sim \frac{Q_{b}}{1+\gamma}$.

The departure process from the load-dependent station $\left(L_{d}\right)$, corresponding to the vehicle network, is studied as a Markov renewal process, and the mean and SCV of the transaction inter-departure times from the vehicle queuing network $\left(\lambda_{d_{t}}^{-1}\right.$ and $\left.c_{d_{t}}^{2}\right)$ are obtained by analyzing the Markov chain embedded at container departure instants from $L_{d}$. Since we approximate the inter-arrival times to Buffer $B_{1}$ with a Coxian 2-phase distribution and the load-dependent service times are exponential random variables, the transition matrix, $P_{D}$ has a special structure. We exploit the structure of this matrix, to determine the steady state stationary probability vector $\left(\Pi_{D}\right)$ and the SCV of the inter-departure times, $c_{d_{t}}^{2}$. (The details of estimating the higher moments are explained in Roy [2011]).

$$
\begin{aligned}
c_{d_{t}}^{2} & =\frac{E\left[T_{D}^{2}\right]-E\left[T_{D}\right]^{2}}{E\left[T_{D}\right]^{2}} \\
c_{d_{t_{u}}}^{2} & =q_{o} c_{d_{t}}^{2}+1-q_{o}
\end{aligned}
$$

where $q_{o}=\frac{\lambda_{a_{t_{u}}}}{\lambda_{a_{t_{u}}}+\lambda_{a_{t_{l}}}}$ 


\section{Model for the ASCs}

Each ASC resource is modeled as a $G I / G / 1$ queue with two classes of transaction requests: loading and unloading containers. Note that each ASC maintains a single virtual queue to process the transactions. While the inter-arrival times for loading transactions to an ASC are exponential with mean $\lambda_{a_{s_{l}}}^{-1}(i)$, the inter-arrival times for unloading containers to the ASCs have a general distribution with parameters $\lambda_{a_{s_{u}}}^{-1}(i)$ and $c_{a_{s_{u}}}^{2}(i)$. Since, each ASC processes both loading and unloading, the expected service times for loading (retrieving) and unloading (stacking) containers are determined as follows. We first consider the case of storing the import containers in the stack during unloading operations. Two cases arise depending on the originating position of the ASC. Let $p_{b}=\frac{\lambda_{a_{s_{l}}}(i)}{\lambda_{a_{s_{l}}}(i)+\lambda_{a_{s_{u}}}(i)}$ and $p_{i}=\frac{\lambda_{a_{u}}(i)}{\lambda_{a_{s_{l}}}(i)+\lambda_{a_{s_{u}}}(i)}$ denote the probabilities of the ASC originating from the buffer location and the interior storage location, respectively. In case 1, the ASC travels from the stack buffer location to the stack location to store the container. If $\left(x_{l_{i}}, y_{m_{i}}\right)$ and $\left(x_{l_{j}}, y_{m_{j}}\right)$ denote the origin and the destination location of the ASC, and $v_{s_{x}}$ and $v_{s_{y}}$ denote the speed of the trolley and the gantry crane, respectively, the travel time is expressed by $\left\{\max \left(\frac{\left|x_{l_{i}}-x_{l_{j}}\right|}{v_{s_{x}}}, \frac{\left|y_{m_{i}}-y_{m_{j}}\right|}{v_{s_{y}}}\right)\right\}$. However, in case 2, the dwell point of the ASC is at the interior of the stacks. So that it first has to retrieve the container from the buffer location and then travel back to store the container in the racks. Let the storage location coordinates be denoted as $\left(x_{l_{k}}, y_{m_{k}}\right)$. Then the travel time for case 2 is given by $\left\{\max \left(\frac{\left|x_{l_{i}}-x_{l_{j}}\right|}{v_{s_{x}}}, \frac{\left|y_{m_{i}}-y_{m_{k}}\right|}{v_{s_{y}}}\right)+\max \left(\frac{\left|x_{l_{k}}-x_{l_{j}}\right|}{v_{s_{x}}}, \frac{\left|y_{m_{k}}-y_{m_{j}}\right|}{v_{s_{y}}}\right)\right\}$. The movement of the ASC storing and retrieving containers in the stack is illustrated in Figure 6.

The container pick-up and drop-off time denoted by $L_{t}^{s}$ and $U_{t}^{s}$, take into account the vertical travel time of the crane. The service time for the ASCs for loading the containers is a mixture of the two service times (the random variables corresponding to the two cases are 
denoted by $X_{s_{l}}$ and $X_{s_{u}}$ respectively). The expected service time, $\mu_{s_{l}}^{-1}(i)$, which depends upon the travel trajectory of the crane, is given by Equation 37 (see Appendix B).

We now develop the expected service time expression for the case of retrieving containers from the stack (for loading containers onto a vessel). Similar to the case of unloading operations, there are two cases depending on the dwell point of the ASC. In the first case, the ASC is present at the buffer location and has to travel to the stack retrieval location and transport the container to the destination buffer location. However, if the ASC is present at an interior stack location, it has to travel to the retrieval stack location and transport the container to the destination buffer location. If the retrieval location coordinates are denoted by $\left(x_{l_{k}}, y_{m_{k}}\right)$, the expected service time, $\mu_{s_{u}}^{-1}(i)$, which depends upon the travel trajectory of the crane, is given by Equation 38 (see Appendix B). In a similar fashion, the second moment of the service time can be calculated by the expression, $p_{b}\left(E\left[X_{s_{l}}^{2}\right]\right)+p_{i}\left(E\left[X_{s_{u}}^{2}\right]\right)$.

Since the arrival rate for stacking containers is uniform for unloading transactions, the arrival rate to a stack crane $i, \lambda_{a_{s_{u}}}(i)$ is given by $\frac{\lambda_{a}^{u}}{N_{s}}$ (Equation 13). Further, using the splitting process idea, the SCV of the inter-arrival times for unloading transactions, $c_{a_{s_{u}}}^{2}(i)$, to each stack crane is given by Equations 14 and 15). Each stack crane is modeled as a single server station with an aggregated class entity (see Bitran and Tirupati [1988] and Satyam and Krishnamurthy [2008] for the expressions to determine expected throughput time and SCV of the inter-departure times for a multi-class single station open queue). The performance measures, such as ASC utilization $\left(\mathbb{U}_{s_{i}}\right)$, expected container waiting times, and throughput times at the cranes for unloading and loading containers, $\left(\mathbb{W}_{s}^{u}(i)\right.$ and $\left.\mathbb{W}_{s}^{l}(i)\right)$, and $\left(\mathbb{E}\left[T_{s}^{u}\right](i)\right.$ and $\left.\mathbb{E}\left[T_{s}^{l}\right](i)\right)$ are obtained using a similar approach adopted for the quayside. Hence, to obtain the SCV of the inter-departure times for the loading transactions at a station $i, c_{d_{s_{l}}}^{2}(i)$, we invoke the SCV splitting equation on the SCV of the inter-departure times for the aggregated class (Equation 16). The SCV of the inter-departure times for 
the aggregated class, $c_{d_{s}}^{2}(i)$, is in turn obtained using an expression similar to Equation 5 , defined for the quayside.

The aggregated SCV of the inter-departure times from all ASC resources for the loading transactions, $c_{a_{t_{l}}}^{2}$, forms the input (SCV of the inter-arrival times) to the vehicle network (Equation 17).

$$
\begin{aligned}
\lambda_{a_{s_{u}}}(i) & =\frac{\lambda_{a}^{u}}{N_{s}} \\
c_{a_{s_{u}}}^{2} & =c_{d_{t_{u}}}^{2} \\
c_{a_{s_{u}}}^{2}(i) & =c_{a_{s_{u}}}^{2} \frac{1}{N_{s}}+\left(1-\frac{1}{N_{s}}\right) \\
c_{d_{s_{l}}}^{2}(i) & =q_{o} c_{d_{s}}^{2}(i)+1-q_{o} \\
c_{a_{t_{l}}}^{2} & =\frac{\sum_{i=1}^{N_{s}} \lambda_{a_{s_{l}}}(i) c_{d_{s_{l}}}^{2}(i)}{\lambda_{a}^{l}}
\end{aligned}
$$

where $i=1, \ldots, N_{s}$ and $q_{o}=\frac{\lambda_{a_{s_{l}}}(i)}{\lambda_{a_{s_{l}}}(i)+\lambda_{a_{s_{u}}}(i)}$.
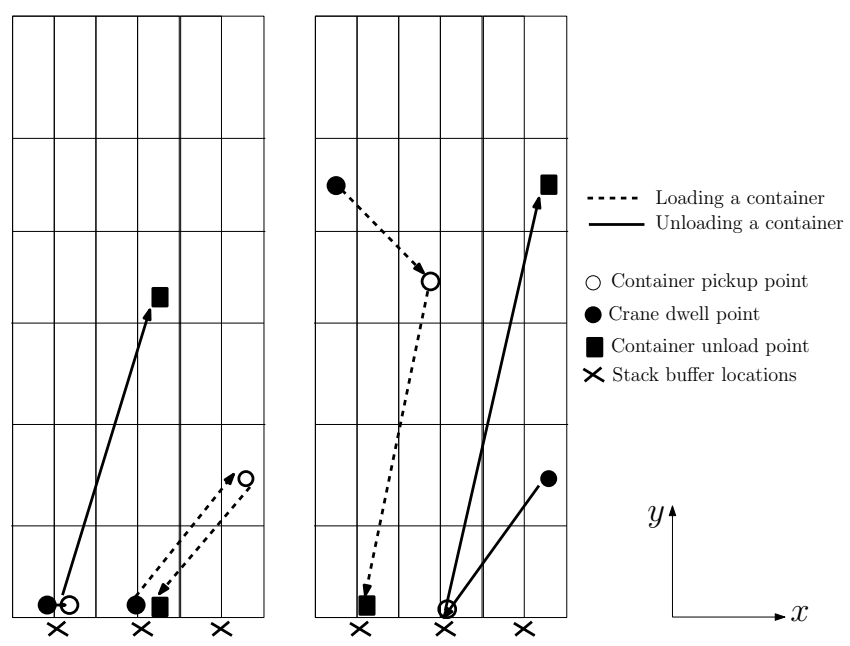

Figure 6: Illustration of ASC travel path for processing loading and unloading operations 


\section{Integrated Analytical Model for Overlapping Operations}

The models for the QCs, ALV movement and ASCs were developed in Sections 4, 5, and 6 respectively. In this Section, we integrate these three models and develop an integrated model for the overlapping loading and unloading operations of a container terminal (Figure 7).

The nodes are connected using departure and arrival process information. In the model, the first and second moment information of the inter-departure times from the $\mathrm{QC}$ resources processing unloading transactions form the inter-arrival time information for the unloading transactions to the vehicle network. Likewise, the first and second moment information of the inter-departure times from the ASC resources processing loading transactions form the inter-arrival time information for the loading transactions to the vehicle network. Using these two pieces of information, the arrival process information to the vehicle network for processing unloading and loading transactions is complete. Now, the first and second moment of the inter-departure times from the vehicle network for the unloading transactions forms the inter-arrival time information for the unloading transactions for the ASC resources. Likewise, the first and second moment of the inter-departure times from the vehicle network for the loading transactions forms the inter-arrival time information for the loading transactions for the QC resources. However, to evaluate the network, the second moment information for the inter-arrival times of the loading transactions at the vehicle network and the inter-arrival times of the unloading transactions at the ASC queues are not available a priori. Hence, we develop an iterative convergence algorithm (IC) to estimate

the second moments of the inter-arrival times and evaluate the network performance. The complete procedure to evaluate the queuing network model is included in Algorithm 1.

We now explain the steps of the solution algorithm. The solution approach begins with 
the initialization of the input parameters $\left(\lambda_{a_{q_{u}}}^{-1}(i)\right.$ and $\left.c_{a_{q_{u}}}^{2}(i)\right)$ to evaluate the performance of the $\mathrm{QC}$ resources processing unloading requests using $G I / G / I$ queues. First, we determine the performance measures and the departure process information from the individual QCs processing unloading requests, $c_{d_{q u}}^{2}(i)$. Then, we use this information to determine the aggregated SCV of the inter-arrival times for the unloading requests from the $\mathrm{QCs}, c_{a_{t_{u}}}^{2}$. To evaluate the reduced vehicle queuing network, the SCV of the inter-arrival times for the aggregated loading requests, $c_{a_{t_{l}}}^{2}$, is also required. Since we do not have this information, we use an iterative procedure to arrive at the appropriate values. We first initialize $c_{a_{t_{l}}}^{2}$ to take a value of 1.0 (also referred as $c_{a_{t_{l}}}^{2}($ curr $)$. We then evaluate the vehicle subnetwork and determine the SCV of inter-departure times, $c_{d_{t}}^{2}$, using an approximate departure process analysis. Then using the splitting process, we determine the SCV of the inter-departure times for the unloading requests, $c_{d_{t_{u}}}^{2}$, which forms the input for evaluating the performance of ASC resources. The ASC network is evaluated using the approach described in Section 6 and the aggregated SCV of the inter-departure times for the loading requests is determined.

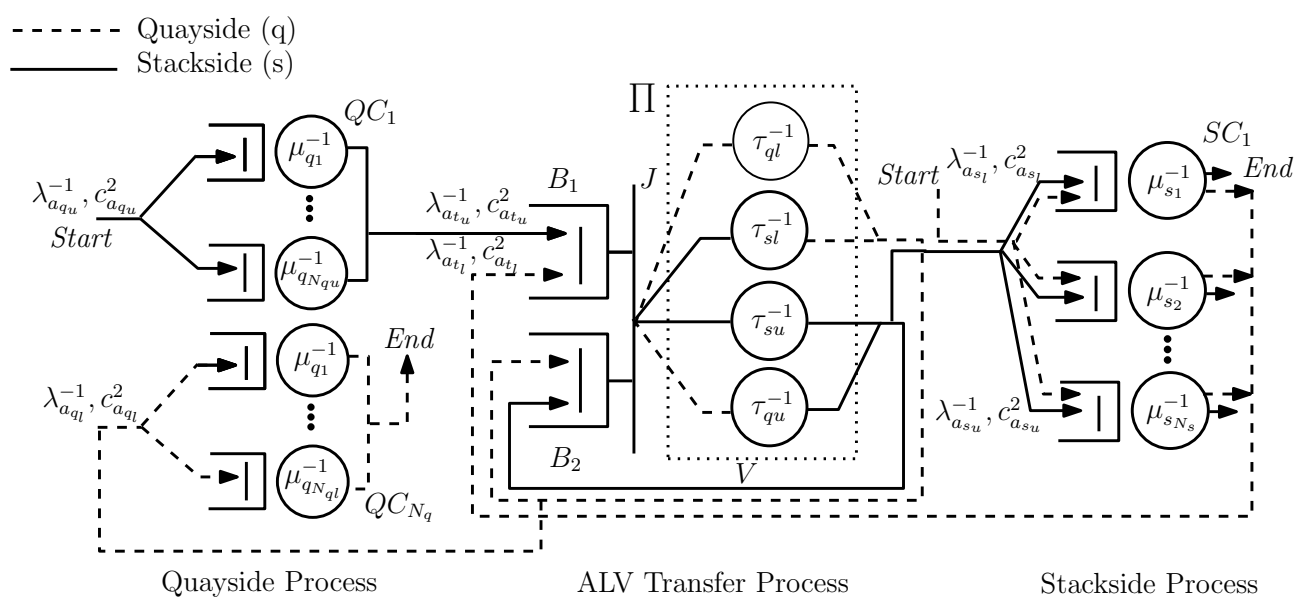

Figure 7: Queuing network model of overlapping operations 
The error component, $\left|c_{a_{l}}^{2}(c u r r)-c_{a_{t_{l}}}^{2}\right|$ is estimated. If the error exceeds the limit, then the vehicle network is re-evaluated using the new estimate of $c_{a_{t_{l}}}^{2}$. Steps 8-12 in the algorithm are repeated until the error component becomes acceptable. Then, the performance measures of the vehicle transport process is evaluated using a CTMC with Cox 2-phase inputs. Finally, the performance of the QC resources processing loading requests is estimated using the SCV of the inter-departure times for the loading requests from the vehicle network $\left(c_{d_{t}}^{2}\right)$.

The expected throughput times for processing a loading and an unloading request, $\mathbb{E}\left[T_{l}\right]$ and $\mathbb{E}\left[T_{u}\right]$, are given by Equations 18 and 19, respectively.

$$
\begin{aligned}
\mathbb{E}\left[T_{l}\right] & =\mathbb{E}\left[T_{q}^{l}\right]+\mathbb{E}\left[T_{t}^{l}\right]+\mathbb{E}\left[T_{s}^{l}\right] \\
\mathbb{E}\left[T_{u}\right] & =\mathbb{E}\left[T_{q}^{u}\right]+\mathbb{E}\left[T_{t}^{u}\right]+\mathbb{E}\left[T_{s}^{u}\right]
\end{aligned}
$$

The next section describes the numerical experiments and model validation results.

\section{Numerical Experiments}

We validate the results obtained from the queuing network model using a detailed simulation model built using AutoMod ${ }^{\complement}$ software v12.2.1 (www.automod.com). For each scenario, 15 replications are run with a warmup period of $24 \mathrm{hrs}$ and a run time of $480 \mathrm{hrs}$ (from about $86400(0.05 \times 3600 \times 24 \times 20)$ to 103680 combined container loading and unloading requests per simulation run), leading to $95 \%$ confidence intervals of the performance measures that lie within $\pm 2 \%, \pm 5 \%$, and $\pm 15 \%$ around the average for utilization, throughput time and queue length measures, respectively. The warmup period eliminates any initial bias due to system startup conditions such as the starting location of the vehicles and cranes. The 3D 
simulation model captures the vehicle movement along the guide-paths and coordination delays at the quayside and at the stackside. The analytical model is executed using Matlab software. The IC algorithm always converged between 30-35 iterations.

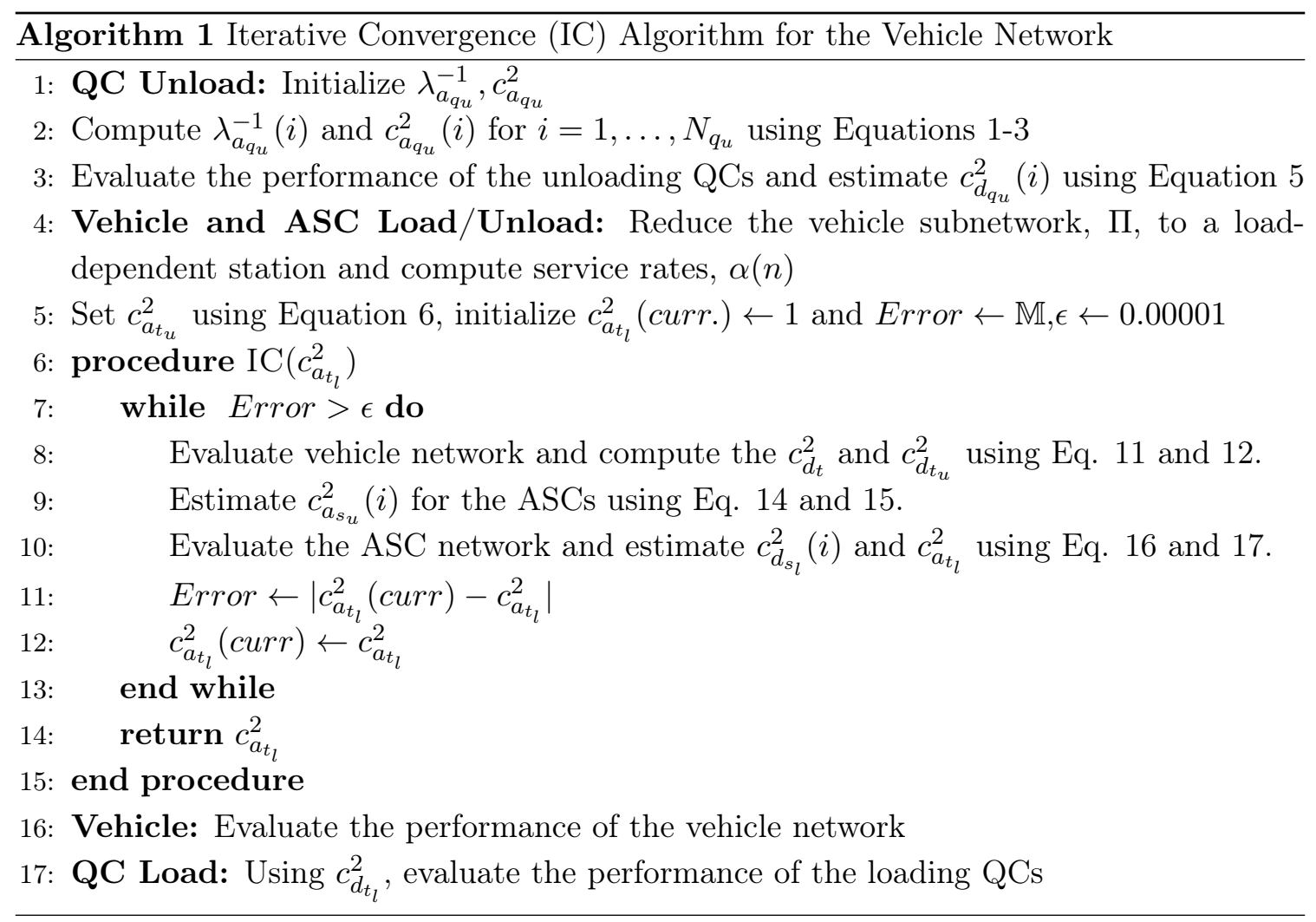

We use an elaborate design of experiments to test the model at different levels of resource parameters. Each level of a resource parameter is associated with a level of utilization: Heavy and Non-heavy. The QC rate is varied at two levels: 35 (heavy) and 45 (non-heavy) cycles per hour; the number of ALVs is varied at two levels: 12 (heavy) and 15 (non-heavy); the number of stack blocks is varied at two levels: 14 (heavy) and 20 (non-heavy). In the case of 20 stack blocks, each stack has 40 bays, whereas in the case of 14 stack blocks, each stack has 57 bays, while the storage capacity is kept constant (Table 5). In Table 6, we list 
the eight experiment categories that we adopt in this research.

Table 5: Design of experiments for model validation (Input) (from De Koster et al. [2004] and personal communication with ECT and APMT, Rotterdam staff)

\begin{tabular}{|c|c|c|}
\hline Quayside & Vehicle Transport & Stackside \\
\hline 6 Quay cranes & Area of $540 \mathrm{~m} \times 90 \mathrm{~m}$, & 24000 storage positions, 20 and 14 stacks, 5 buffer lanes/ stack \\
\hline capacity: 35 and 45 cycles $/ \mathrm{hr}$ & 12 and $15 \mathrm{ALVs}$ & each stack has 6 rows and 5 tiers \\
\hline 4 buffer lanes, & velocity: $6 \mathrm{~m} / \mathrm{s}$ & velocity: $3 \mathrm{~m} / \mathrm{s}$ \\
\hline
\end{tabular}

Table 6: Experiment categories

\begin{tabular}{crrrc}
\hline Number & QC Traffic & ALV Traffic & ASC Traffic & Arrival Rate (per hour) \\
\hline 1 & Non-heavy & Non-heavy & Non-heavy & $180-216$ \\
2 & Non-heavy & Non-heavy & Heavy & $191-212$ \\
3 & Non-heavy & Heavy & Non-heavy & $180-198$ \\
4 & Non-heavy & Heavy & Heavy & $191-202$ \\
5 & Heavy & Non-heavy & Non-heavy & $180-198$ \\
6 & Heavy & Non-heavy & Heavy & $194-202$ \\
7 & Heavy & Heavy & Non-heavy & $180-198$ \\
8 & Heavy & Heavy & Heavy & $194-202$ \\
\hline
\end{tabular}

For each experiment number, the container arrival rate is varied at 10 equidistant levels such that the resource utilization criteria are met, see Table 6. If the resource utilization exceeds $85 \%$, we denote the traffic level as 'heavy', otherwise we denote it as 'non-heavy.' Note that we have three subnetworks (resources) here, QC, ALV, and ASC. Therefore, for a particular container arrival rate, none, some, or all resources will be subjected to heavy traffic depending on the resource service rate. In total, we analyze 80 scenarios using both the detailed simulation model as well as the analytical model. As an example, for experiment 8, the 10 scenarios are generated for heavy traffic levels (the QC utilizations vary between $92 \%$ and $96 \%$, the ALV utilizations vary between $90 \%$ and $94 \%$, and the ASC utilizations vary between $85 \%$ and $89 \%$ ).

The histograms with the percentage absolute error distribution for all measures are 
shown in Appendix C (Figures C.1-C.6). The average absolute error percentage in expected load and unload throughput time $\left(\mathbb{E}\left[T_{l}\right]\right.$ and $\left.\mathbb{E}\left[T_{u}\right]\right)$, QC utilization $\left(\mathbb{U}_{q}\right)$, ASC utilization $\left(\mathbb{U}_{s}\right)$, vehicle utilization $\left(\mathbb{U}_{v}\right)$, and number of containers (loading and unloading) waiting for the QC $\left(\mathbb{L}_{q}^{l}\right.$ and $\left.\mathbb{L}_{q}^{u}\right)$, and ASC $\left(\mathbb{L}_{s}^{l}\right.$ and $\left.\mathbb{L}_{s}^{u}\right)$ are obtained by the expression $\left(\left|\frac{A-S}{S}\right| \times 100\right)$, where $A$ and $S$ correspond to the estimate of the measures obtained from the analytical and simulation model, respectively.

Table 7 summarizes the results. For all resources, the averages are calculated over those experiments where the utilization of the resource is either 'heavy' or 'non-heavy.' The average errors in all performance measures (except $\mathbb{L}_{t}^{l}$ and $\mathbb{L}_{t}^{u}$ ) are moderate. The average errors in resource utilizations are less than $1 \%$, whereas the throughput time and the queue length estimates are less than $12 \%$ and $20 \%$, respectively. We also observe that the stack crane performance measure approximations, which are modeled using Whitt's two-moment approximation (and correction factor for heavy traffic), are accurate.

Table 7: Average absolute errors for the performance measures

\begin{tabular}{rcccccccccccccc}
\hline & $\mathbb{U}_{q}^{l}$ & $\mathbb{E}\left[T_{q}^{l}\right]$ & $\mathbb{L}_{q}^{l}$ & $\mathbb{U}_{q}^{u}$ & $\mathbb{E}\left[T_{q}^{u}\right]$ & $\mathbb{L}_{q}^{u}$ & $\mathbb{U}_{t}$ & $\mathbb{E}\left[T_{t}^{l}\right]$ & $\mathbb{E}\left[T_{t}^{u}\right]$ & $\mathbb{L}_{t}^{l}$ and $\mathbb{L}_{t}^{u}$ & $\mathbb{U}_{s}$ & $\mathbb{E}\left[T_{s}^{l}\right]$ & $\mathbb{E}\left[T_{s}^{u}\right]$ & $\mathbb{L}_{s}^{u}$ and $\mathbb{L}_{s}^{l}$ \\
\hline Heavy & $0.2 \%$ & $7.1 \%$ & $11.1 \%$ & $0.2 \%$ & $2.3 \%$ & $4.2 \%$ & $0.2 \%$ & $8.4 \%$ & $11.3 \%$ & $46.3 \%$ & $0.5 \%$ & $1.3 \%$ & $1.4 \%$ & $5.1 \%$ \\
Non-heavy & $0.2 \%$ & $9.2 \%$ & $19.9 \%$ & $0.3 \%$ & $0.4 \%$ & $1.4 \%$ & $0.2 \%$ & $1.1 \%$ & $2.5 \%$ & - & $0.7 \%$ & $0.3 \%$ & $0.8 \%$ & $3.3 \%$ \\
\hline
\end{tabular}

The average errors for the combined container queue waiting for availability of ALVs (sum of $\mathbb{L}_{t}^{l}$ and $\mathbb{L}_{t}^{u}$ ) in heavy traffic level is rather high (up to 46\%). This error is large, but not unusual for heavy traffic situations (85\%-95\%) utilizations, due to strong non-linear growth of queues at such high utilizations (Jia and Heragu [2009], Roy [2011]). Note that we reduce the vehicle network to a load-dependent station. The most challenging part in evaluating the load-dependent queue is parameterizing the coefficient of variation of the load-dependent service times (Tolopka [1979]).

We observe that the average errors in total unload and load throughput time, which 
are the measures of interest of this study, are less than $1.9 \%$ and $3.4 \%$, with the maximum errors of $6.3 \%$ and $9.5 \%$, respectively (see Figures C.8 and C.7 for distribution of errors). Hence, we are confident that our model gives sufficiently accurate estimates of container throughput times.

\section{Design Insights: Efficient Stack Layout}

We investigate the effect of different terminal configurations on the performance of the overlapping operations and identify good configurations that provide a significant performance advantage. We keep the container arrival rate constant for loading and unloading containers at $90 / \mathrm{hr}$, and vary the stack configuration. The number of stack blocks $\left(N_{s}\right)$, the number of rows per stack block $\left(N_{r}\right)$, and the number of tiers per stack block $\left(N_{t}\right)$ are varied but the number of storage locations is fixed at 24000. $N_{s}$ is varied between 10 and 120 with increments of $5, N_{r}$ is varied from 4 to 10 with increments of 2 , and $N_{t}$ has values 3 or 5 . The fleet size is 40 . QCs 1,5 , and 6 load containers to the vessel while QCs 2,3 , and 4 unload containers from the vessel. Some configurations appear to be unstable and have extremely high throughput times due to high stack crane utilization or high vehicle utilization. Table 8 shows the best five design choices for unloading operations only, whereas Table 9 shows the best five design choices for both loading and unloading operations. In both tables, we see that a large number of stack blocks and a large number of bays per stack block are not preferable for better throughput time performance. We also find that there are common design profiles that maximize the terminal performance for both single as well as overlapping operations. For instance, in this case, three out of five design profiles are common to both types of operations. Design profiles such as $N_{S}=15, N_{r}=10, N_{b}=32$, $N_{t}=5$ and $N_{S}=20, N_{r}=10, N_{b}=24, N_{t}=5$ are top performers. The throughput 
times between the first and the fifth design configuration differ by about $5 \%$ in both single and overlapping operations. However, the expected throughput time for the three common configurations in the case of single operation differs by a maximum of $17 \%$ compared to the throughput time in the corresponding stack design in the case of overlapping operations (by assigning a weight of $50 \%$ each to loading and unloading operations).

\section{Conclusions}

This research is an initial attempt to develop an analytical model of an integrated container terminal with overlapping operations. The model captures the stochastic interactions among quayside, vehicle transport and stackside resources. The quayside and stackside resources are modeled using single-server queues, whereas the vehicle transport process is modeled using a multi-class semi-open queuing network with class switching. Integrating the vehicle movement submodel with the QC and the ASC submodels is complex because the higher moments of the inter-departure times from the vehicle transport network are unavailable. We address this complexity by first reducing the vehicle queuing network to a single-chain semi-open queuing network with a single load-dependent station. We approximate the higher moments of the inter-departure times from the vehicle network using embedded Markov chain analysis, and link the vehicle, ASC, and the QC models using an iterative convergence algorithm.

Using detailed simulations, we validate the analytical model at both heavy and nonheavy traffic conditions and also develop design insights. We show that there are good stack layout configurations common to both overlapping and single operations, i.e., good stack designs for single operations (in the top five) do not vary more than $17 \%$ in expected throughput times compared to the best designs for overlapping operations. 
Table 8: Good terminal layout design choices with only unloading operations

\begin{tabular}{rrrrrrrrrrr}
\hline$N_{s}$ & $N_{r}$ & $N_{b}$ & $N_{t}$ & $\mathbb{U}_{q}$ & $\mathbb{E}\left[T_{q}\right](\mathrm{sec})$ & $\mathbb{U}_{t}$ & $\mathbb{E}\left[T_{t}\right](\mathrm{Sec})$ & $\mathbb{U}_{s}$ & $\mathbb{E}\left[T_{s}\right](\mathrm{Sec})$ & $\mathbb{E}\left[T_{u}\right](\mathrm{sec})$ \\
\hline 20 & 10 & 24 & 5 & $42 \%$ & 137.3 & $15.3 \%$ & 245.5 & $19.0 \%$ & 187.0 & 569.8 \\
25 & 8 & 24 & 5 & $42 \%$ & 137.3 & $16.4 \%$ & 262.8 & $15.2 \%$ & 178.6 & 578.8 \\
20 & 8 & 30 & 5 & $42 \%$ & 137.3 & $13.9 \%$ & 222.7 & $22.0 \%$ & 225.4 & 585.5 \\
25 & 10 & 20 & 5 & $42 \%$ & 137.3 & $18.3 \%$ & 292.0 & $13.6 \%$ & 156.8 & 586.1 \\
30 & 8 & 20 & 5 & $42 \%$ & 137.3 & $19.0 \%$ & 304.1 & $11.4 \%$ & 152.8 & 594.2 \\
\hline
\end{tabular}

Table 9: Good terminal layout design choices with both loading and unloading operations

\begin{tabular}{|c|c|c|c|c|c|c|c|c|c|c|c|c|c|c|c|}
\hline$N_{s}$ & $N_{r}$ & $N_{b}$ & $N_{t}$ & $\mathbb{U}_{q}$ & $\mathbb{E}\left[T_{q}^{l}\right](\mathrm{Sec})$ & $\mathbb{E}\left[T_{q}^{u}\right](\mathrm{Sec})$ & $\mathbb{U}_{t}$ & $\mathbb{E}\left[T_{t}^{u}\right](\mathrm{sec})$ & $\mathbb{E}\left[T_{t}^{l}\right](\mathrm{Sec})$ & $\mathbb{U}_{s}$ & $\mathbb{E}\left[T_{s}^{u}\right](\mathrm{sec})$ & $\mathbb{E}\left[T_{s}^{l}\right](\mathrm{sec})$ & $\mathbb{E}\left[T_{u}\right](\mathrm{sec})$ & $\mathbb{E}\left[T_{l}\right](\mathrm{sec})$ & $0.5 \mathbb{E}\left[T_{u}\right]+0.5 \mathbb{E}\left[T_{l}\right](\mathrm{sec})$ \\
\hline 15 & 10 & 32 & 5 & $42 \%$ & 141.0 & 126.3 & $12.6 \%$ & 187.1 & 216.3 & $22.3 \%$ & 147.0 & 168.4 & 460.4 & 525.6 & 493.0 \\
\hline 20 & 10 & 24 & 5 & $42 \%$ & 140.9 & 126.3 & $15.6 \%$ & 229.8 & 270.6 & $13.4 \%$ & 109.2 & 125.3 & 465.4 & 536.7 & 501.0 \\
\hline 20 & 8 & 30 & 5 & $42 \%$ & 141.0 & 126.3 & $14.2 \%$ & 209.1 & 244.8 & $15.9 \%$ & 131.9 & 152.0 & 467.4 & 537.8 & 502.6 \\
\hline 25 & 8 & 24 & 5 & $42 \%$ & 140.8 & 126.3 & $16.7 \%$ & 244.1 & 289.2 & $10.7 \%$ & 106.9 & 122.9 & 477.4 & 552.9 & 515.2 \\
\hline 15 & 8 & 40 & 5 & $42 \%$ & 140.9 & 126.3 & $11.6 \%$ & 172.3 & 198.1 & $26.7 \%$ & 184.1 & 210.7 & 482.8 & 549.8 & 516.3 \\
\hline
\end{tabular}

\section{References}

H. Bae, R. Choe, T. Park, and K. Ryu. Comparison of operations of AGVs and ALVs in an automated container terminal. Journal of Intelligent Manufacturing, 22(3):413-426, 2011.

F. Baskett, K. M. Chandy, R. R. Muntz, and F. G. Palacios. Open, closed, and mixed networks of queues with different classes of customers. J. ACM, 22(2):248-260, 1975.

E. K. Bish. A multiple-crane-constrained scheduling problem in a container terminal. European Journal of Operational Research, 144:83, 2003.

G. R. Bitran and D. Tirupati. Multiproduct queueing networks with deterministic routing: Decomposition approach and the notion of interference. Management Science, 34(1): 75-100, 1988.

B. Brinkmann. Operations systems of container terminals: A compendious overview. Springer, Berlin/Heidelberg, 2010. 
D. Briskorn, A. Drexl, and S. Hartmann. Inventory-based dispatching of automated guided vehicles on container terminals. In K.H. Kim and Hans-Otto Günther, editors, Container Terminals and Cargo Systems, pages 195-214. Springer Berlin Heidelberg, 2007. doi: 10.1007/978-3-540-49550-5_10.

P. Canonaco, P. Legato, R.M. Mazza, and R. Musmanno. A queuing network model for the management of berth crane operations. Comput. Oper. Res., 35(8):2432-2446, 2008.

K. M. Chandy, U. Herzog, and L. Woo. Parametric analysis of queuing networks. IBM Journal of Research and Development, 19(1):36-42, 1975.

M.B.M. De Koster, T. Le-Anh, and R. J. van der Meer. Testing and classifying vehicle dispatching rules in three real-world settings. Journal of Operations Management, 22(4): $369-386,2004$.

M. Duinkerken, R. Dekker, S. Kurstjens, J. Ottjes, and N. Dellaert. Comparing transportation systems for inter-terminal transport at the Maasvlakte container terminals. In K.H. Kim and Hans-Otto Günther, editors, Container Terminals and Cargo Systems, pages 37-61. Springer Berlin Heidelberg, 2007.

E. D. Edmond and R. P. Maggs. How Useful are Queue Models in Port Investment Decisions for Container Berths? The Journal of the Operational Research Society, 29(8):741-750, 2009.

A.H. Gharehgozli, G. Laporte, Y. Yu, and R. De Koster. Scheduling twin yard cranes in a container block. Transportation Science, 2014a.

A.H. Gharehgozli, D. Roy, and R. De Koster. Sea container terminals: New technologies, or models, and emerging research areas. Technical report, Rotterdam, 2014b. 
Global Industry Analysts Inc. Maritime containerization: A global strategic business report. "http://www.prweb.com/releases/containerization/container_shipping/ prweb9382752.htm", 2013. Accessed: 2013-04-15.

A.V. Goodchild and C.F. Daganzo. Double-cycling strategies for container ships and their effect on ship loading and unloading operations. Transportation Science, 40(4):473-483, 2006.

S. Hoshino, J. Ota, A. Shinozaki, and H. Hashimoto. Optimal design methodology for an agv transportation system by using the queuing network theory. In R. Alami, R. Chatila, and H. Asama, editors, Distributed Autonomous Robotic Systems 6, pages 411-420. Springer Japan, 2007.

J. Jia and S.S. Heragu. Solving semi-open queuing networks. Operations Research, 57(2): 391-401, 2009.

K. H. Kim and K. Y. Kim. An optimal routing algorithm for a transfer crane in port container terminals. Transportation Science, 33:17, 1999.

K. H. Kim and Y. Park. A crane scheduling method for port container terminals. European Journal of Operational Research, 156(3):752 - 768, 2004.

C. Li and G.L. Vairaktarakis. Loading and unloading operations in container terminals. IIE Transactions, 36(4):287-297, 2004.

C. Liang, Y. Huang, and Y. Yang. A quay crane dynamic scheduling problem by hybrid evolutionary algorithm for berth allocation planning. Computers $\&$ Industrial Engineering, 56(3):1021 - 1028, 2009.

F. Meisel. Seaside operations planning in container terminals. Springer, Physica Verlag Heidelberg., 2009. 
W. C. Ng. Crane scheduling in container yards with inter-crane interference. European Journal of Operational Research, 164:64-78, 2005.

Matthew E.H. Petering. Effect of block width and storage yard layout on marine container terminal performance. Transportation Research Part E: Logistics and Transportation Review, 45(4):591 - 610, 2009.

M.E.H. Petering. Development and simulation analysis of real-time, dual-load yard truck control systems for seaport container transshipment terminals. OR Spectrum, 32(3): 633-661, 2010.

M.E.H. Petering, Y. Wu, W. Li, M. Goh, and R. Souza. Development and simulation analysis of real-time yard crane control systems for seaport container transshipment terminals. OR Spectrum, 31(4):801-835, 2009.

D. Roy. Design and Analysis of Unit-load Warehouse Operations using Autonomous Vehicles. PhD thesis, University of Wisconsin-Madison, Department of Industrial and Systems Engineering, Madison, Wisconsin, 2011.

D. Roy and R. De Koster. Modeling and design of container terminal operations. Technical report, Rotterdam, 2012.

K. Satyam and A. Krishnamurthy. Performance evaluation of a multi-product system under conwip control. IIE Transactions, 40(3):252-264, 2008.

R. Stahlbock and S. Voß. Operations research at container terminals: a literature update. OR Spectrum, 30(1):1-52, 2008.

D. Steenken, S. Voß, and R. Stahlbock. Container terminal operation and operations research - a classification and literature review. In Hans-Otto G $\tilde{A} \frac{1}{4}$ nther and K.H. Kim, 
editors, Container Terminals and Automated Transport Systems, pages 3-49. Springer Berlin Heidelberg, 2005.

TBA BV. Design of terminal layout and handling systems. "http://www.freight-int. com/article/design-of-terminal-lay-out-and-handling-systems.html.", 2010. Accessed: 2011-12-26.

S. Tolopka. Solution of general queueing networks using norton's theorem. Technical Report TR-314, West Lafayette, IN, United States, 1979.

I. F. A. Vis and R. De Koster. Transshipment of containers at a container terminal: An overview. European Journal of Operational Research, 147(1):1-16, 2003.

I. F. A. Vis and K. J. Roodbergen. Scheduling of container storage and retrieval. Operations Research, 57(2):456-467, 2009.

I.F.A. Vis and I. Harika. Comparison of vehicle types at an automated container terminal. OR Spectrum, 26(1):117-143, 2004.

W. Whitt. The queueing network analyzer. Bell System Technical Journal, 62(9):2779-2815, 1983.

\section{A Estimating Vehicle Service Times}

The various ALV service types are illustrated using Figure A.1. 

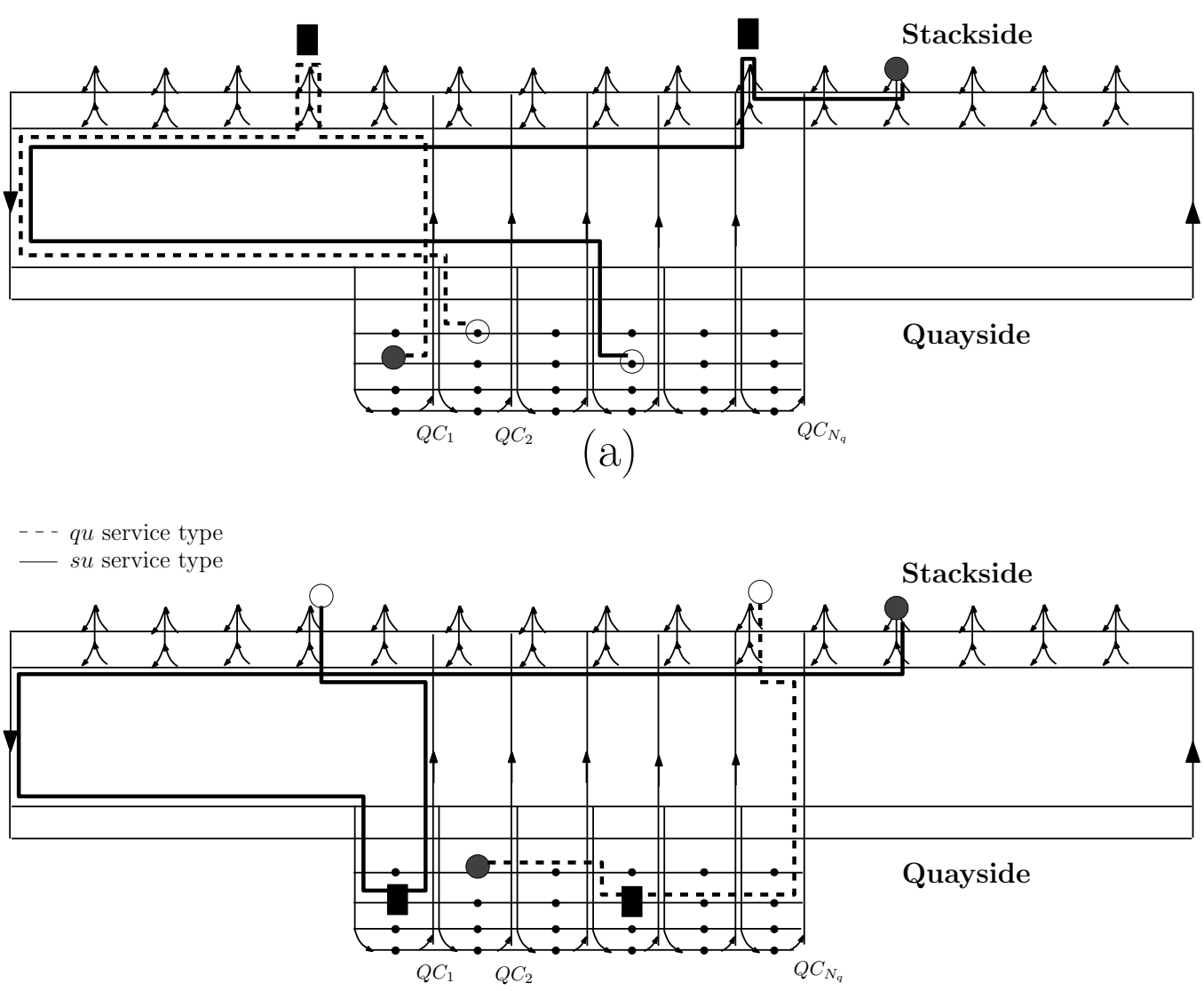

(b)

Figure A.1: Illustration of vehicle service types for processing (a) loading operations and (b) unloading operations

\section{A.1 Service Time Expressions for the Vehicle Service Types}

The expected service time expressions for the four service types are described now. The notations adopted in developing the expected service time expressions for the four service types in the vehicle transport model can be found in Table 2. 
Expected service time expression for the ql service type: In the $q l$ service type, the vehicle's starting position is at the quayside (after unloading a container for the vessel loading process). The vehicle needs to travel to the stackside to pickup another container and to transport it to the quayside. The expected travel times from quayside to the stackside and from stackside to the quayside for loading operations are denoted by $\tau_{q s_{l}}^{-1}$ and $\tau_{s q_{l}}^{-1}$, respectively.

To estimate $\tau_{q s_{l}}^{-1}$, three cases are developed based on the location of the destination stack block $(j)$ relative to the location of the shortcut path corresponding to the QC index $(i)$. In case 1 , the travel time expression is derived when the destination stack block is located to the left of the shortcut path $S P_{i}$ corresponding to the quay crane, $Q C_{i}$. In case 2 , the destination stack block is located between the shortcut path $S P_{i}$ corresponding to the quay crane $Q C_{i}$ and $Q C_{N_{q}}$. Finally, in case 3, the destination stack block is located to the right of the last shortcut path corresponding to $Q C_{N_{q}}$. The number of possible scenarios for travel between $N_{q_{l}}$ QCs and $N_{s}$ ASCs is given by $N_{q_{l}} \times N_{s}$. For purpose of illustration, we explain the derivation of the service time expression corresponding to the case 1 where $i=1, \ldots, N_{q_{l}}$, and $j=1, \ldots, N_{s}-N_{s r}\left(\mathbb{S}_{q_{l}}(i)\right)$. The subexpression $\frac{D_{e x}}{2 h_{v}}+\frac{W_{b l}+\left(\left(N_{b_{q}}-1\right) W_{b q}\right) / 2}{h_{v}}$ denotes the vehicle travel at the quayside buffer area. The subexpression $\frac{W_{l}}{h_{v}}$ denotes vertical travel time along the shortcut path (y-axis); the subexpression $\frac{W_{b s}}{2 h_{v}}+\left(N_{s}-N_{s r}\left(\mathbb{S}_{q_{l}}(i)\right)-\right.$ j) $\left(\frac{W_{s}+W_{b s}}{h_{v}}\right)+\frac{W_{s}}{2 h_{v}}$ denotes the travel time along the stackside area to reach the destination stack $j$ from the QC index $\mathbb{S}_{q_{l}}(i)$. The final travel time component $\frac{D_{s l}}{h_{v}}$ denotes the travel time in the buffer lane at the destination stack block $j$. 


$$
\begin{aligned}
& \text { Case 1: } \tau_{q s_{l}}(i, j)^{-1}=\frac{W_{b l}+\left(\left(N_{b_{q}}-1\right) W_{b q}\right) / 2}{h_{v}}+\frac{D_{e x}}{2 h_{v}}+\frac{W_{l}}{h_{v}} \\
& +\left(N_{s}-N_{s r}\left(\mathbb{S}_{q_{l}}(i)\right)-j\right)\left(\frac{W_{s}+W_{b s}}{h_{v}}\right)+\frac{W_{s}+W_{b s}}{2 h_{v}}+\frac{D_{s l}}{h_{v}} \\
& \text { for } i=1, \ldots, N_{q_{l}}, j=1, \ldots, N_{s}-N_{s r}\left(\mathbb{S}_{q_{l}}(i)\right) \\
& \text { Case 2: } \tau_{q s_{l}}(i, j)^{-1}=\frac{W_{b l}+\left(\left(N_{b_{q}}-1\right) W_{b q}\right) / 2}{h_{v}}+\frac{D_{e x}}{2 h_{v}}+\left(j-\left(N_{s}-N_{s r}(i)\right)\right)\left(\frac{D_{i n}+D_{e x}}{h_{v}}\right) \\
& +\frac{W_{l}}{h_{v}}+\frac{W_{s}+W_{b s}}{2 h_{v}}+\frac{D_{s l}}{h_{v}} \\
& \text { for } i=1, \ldots, N_{q_{l}}, \mathbb{S}_{q_{l}}(i) \neq N_{q} \text {, } \\
& j=N_{s}-N_{s r}\left(\mathbb{S}_{q l}(i)\right)+1, \ldots, N_{s}-N_{s r}\left(\mathbb{S}_{q l}(i)\right)+N_{s q}\left(\mathbb{S}_{q_{l}}(i)\right)-1 \\
& \text { Case 3: } \tau_{q s_{l}}(i, j)^{-1}=\frac{W_{b l}+\left(\left(N_{b_{q}}-1\right) W_{b q}\right) / 2}{h_{v}}+\frac{D_{e x}}{2 h_{v}}+\left(N_{s q}\left(\mathbb{S}_{q_{l}}(i)\right)-1\right)\left(\frac{D_{i n}+D_{e x}}{h_{v}}\right)+\frac{L_{r}}{h_{v}}+\frac{W_{l}}{h_{v}} \\
& +\left(N_{s}-j\right)\left(\frac{W_{s}+W_{b s}}{h_{v}}\right)+\frac{D_{e}}{h_{v}}+\frac{W_{s}}{2 h_{v}}+\frac{D_{s l}}{h_{v}} \\
& \text { for } i=1, \ldots, N_{q_{l}}, j=N_{s}-N_{s r}\left(\mathbb{S}_{q_{l}}(i)\right)+N_{s q}\left(\mathbb{S}_{q_{l}}(i)\right), \ldots, N_{s} \\
& \tau_{q s_{l}}^{-1}=\sum_{i=1}^{N_{q_{l}}} \sum_{j=1}^{N_{s}} \frac{1}{\left(N_{q_{l}} N_{s}\right)} \tau_{q s_{l}}(i, j)^{-1}
\end{aligned}
$$

Similarly, expected vehicle travel time from stackside to quayside is provided by Equation 24. The components of expected travel time in Equation 24 are explained now. The travel time expression consists of three travel segments. The first travel segment is along the stack block. It includes traveling from the originating stack block $j$ 's buffer lane to the end of the segment $\left(\frac{W_{s}}{2 h_{v}}+(j-1) \frac{W_{s}+W_{b s}}{h_{v}}\right)$. The second travel segment includes travel along the width of the travel path $\left(\frac{W_{l}}{h_{v}}\right)$. The third travel segment comprises multiple subsegment travel components. The first travel subsegment is to reach the quayside area $\left(\frac{L_{l}}{h_{v}}\right)$. The second is to reach the destination $\mathrm{QC} \mathbb{S}_{q_{l}}(i),\left(\frac{D_{e x}}{2 h_{v}}\right)+\left(\mathbb{S}_{q_{l}}(i)-1\right)\left(\frac{D_{i n}+D_{e x}}{h_{v}}\right)+\left(\frac{D_{s l}+D_{e}}{h_{v}}\right)$, and the final subsegment is to reach the destination buffer lane at the QC $\left(\frac{W_{b l}+\left(\left(N_{b_{q}}-1\right) W_{b q}\right) / 2}{h_{v}}\right)$. 


$$
\begin{aligned}
\tau_{s q_{l}}^{-1} & =\sum_{i=1}^{N_{q_{l}}} \sum_{j=1}^{N_{s}} \frac{1}{\left(N_{q_{l}} N_{s}\right)}\left(\frac{W_{s}}{2 h_{v}}+(j-1) \frac{W_{s}+W_{b s}}{h_{v}}+\frac{W_{l}}{h_{v}}+\frac{L_{l}}{h_{v}}+\right. \\
& \left.+\frac{W_{b l}+\left(\left(N_{b_{q}}-1\right) W_{b q}\right) / 2}{h_{v}}+\frac{D_{e x}}{2 h_{v}}+\left(\mathbb{S}_{q_{l}}(i)-1\right) \frac{D_{i n}+D_{e x}}{h_{v}}+\frac{D_{s l}+D_{e}}{h_{v}}\right)
\end{aligned}
$$

After considering the deterministic container loading and unloading times $\left(L_{t}\right.$ and $\left.U_{t}\right)$ along with the travel time expressions $\tau_{s q_{l}}^{-1}$ and $\tau_{q s_{l}}^{-1}$, we obtain the expected travel time, $\tau_{q l}^{-1}$, for the $q l$ service type (Equation 25).

$$
\tau_{q l}^{-1}=\tau_{q s_{l}}^{-1}+\tau_{s q_{l}}^{-1}+L_{t}^{v}+U_{t}^{v}
$$

Expected service time expression for the su service type: In the su service type, the vehicle's starting position is from the stackside (after unloading the container at the stack buffer position). The vehicle needs to travel to the quayside to retrieve another container (that was unloaded by the QC from the vessel) and transport it to the destination stack block for storage. The expected travel times from stackside to quayside and from quayside to stackside for loading operations are denoted by $\tau_{s q_{u}}^{-1}$ and $\tau_{q s_{u}}^{-1}$, respectively.

$\tau_{q s_{u}}^{-1}$ can be derived in a similar manner by using the constant $N_{q_{u}}$ and the set $\mathbb{S}_{q_{u}}$ instead of $N_{q_{l}}$ and the set $\mathbb{S}_{q_{l}}$, respectively in Equations 20-23. $\tau_{s q_{u}}^{-1}$ can be derived in a similar manner by using the set $N_{q_{u}}$ instead of $N_{q_{l}}$ in Equation 24. Using the expressions for $\tau_{s q_{u}}^{-1}, \tau_{q s_{u}}^{-1}$, and loading/unloading times $\left(L_{t}, U_{t}\right), \tau_{s u}^{-1}$ is expressed using Equation 26.

$$
\tau_{s u}^{-1}=\tau_{s q_{u}}^{-1}+\tau_{q s_{u}}^{-1}+L_{t}^{v}+U_{t}^{v}
$$

Expected service time expression for the qu service type: To estimate $\tau_{q u}^{-1}$, two cases arise. 
In case 1 , the destination unloading $\mathrm{QC}$ is to the right of the originating vehicle's current QC. In this case, the vehicle needs to switch to the appropriate buffer lane and travel to the destination QC buffer lane. On the other hand, if the destination unloading QC is to the left of the vehicle's current QC, the vehicle needs to complete one loop along the rectangular path to reach the destination QC buffer lane due to unidirectional travel path (case 2). Equation 27 provides the expression for expected travel to switch between the origin and the destination buffer lanes. The expressions for the cases 1 and 2 are given by Equations 28 and 29 respectively. Using the two equations, the expression for $\tau_{q q_{u}}^{-1}$ is given by Equation 30 .

$$
\begin{aligned}
& T_{i n}=\sum_{i=1}^{N_{b}} \sum_{j=1}^{N_{b_{q}}} \frac{1}{h_{v} N_{b_{q}}^{2}} W_{b q}|i-j| \\
& \text { Case 1: } \tau_{q q_{u}}(i, j)^{-1}=\left(\frac{D_{e x}+D_{i n}}{h_{v}}\right)\left(\mathbb{S}_{q_{u}}(j)-\mathbb{S}_{q_{u}}(i)\right)+T_{i n} \\
& \text { for } i \in \mathbb{S}_{q_{l}}, j \in \mathbb{S}_{q_{u}}, j \geq i \\
& \text { Case 2: } \tau_{q q_{u}}(i, j)^{-1}=\frac{W_{b l}+\left(\left(N_{b_{q}}-1\right) W_{b q}\right) / 2}{h_{v}}+2 \frac{\left(W_{l}-2 W_{b l}\right)+\left(W_{b s} / 2\right)}{h_{v}} \\
& +\frac{\left(N_{s}-N_{s r}\left(\mathbb{S}_{q_{l}}(i)\right)\right)\left(W_{s}\right)+\left(N_{s}-N_{s r}\left(\mathbb{S}_{q_{l}}(i)\right)-1\right)\left(W_{b s}\right)}{h_{v}} \\
& +\frac{D_{e}+L_{l}+\left(D_{e x} / 2\right)}{h_{v}}+\frac{\left(\mathbb{S}_{q_{l}}(j)-1\right)\left(D_{e x}+D_{i n}\right)}{h_{v}} \\
& +\frac{\left(W_{b l}+\left(N_{b_{q}}-1\right) W_{b q} / 2\right)}{h_{v}} \\
& \text { for } i \in \mathbb{S}_{q_{l}}, j \in \mathbb{S}_{q_{u}}, j<i \\
& \tau_{q q_{u}}^{-1}=\sum_{i=1}^{N_{q_{l}}} \sum_{j=1}^{N_{q_{u}}} \frac{1}{\left(N_{q_{l}} N_{q_{u}}\right)} \tau_{q q_{l u}}(i, j)^{-1}
\end{aligned}
$$

Using the quantities, $\tau_{q q_{u}}^{-1}$ and $\tau_{q s_{u}}^{-1}$, the expected service time expression for the $q u$ service 
type is given by Equation 31 .

$$
\tau_{q u}^{-1}=\tau_{q q_{u}}^{-1}+\tau_{q s_{u}}^{-1}+L_{t}^{v}+U_{t}^{v}
$$

Expected service time expression for the sl service type: In the sl service type, the vehicle travels from one stack block to another to pick up the container and then travels to the quayside to deposit the container at the QC buffer lane. Therefore, we develop two cases to determine the expected travel time of a vehicle to travel from its current stack location to the destination stack location. In case 1, the destination stack block is to the left of the origin stack block. Therefore, the vehicle travels along the stackside travel path to reach the destination stack location $\left(\frac{\left(W_{s}+W_{b s}\right)(i-j)}{h_{v}}\right)$. Travel along the stackside buffer lane at the origin and at the destination location is given by $\frac{2 D_{s l}}{h_{v}}$. In case 2 , the vehicles needs to travel along the rectangular loop to reach the destination stack location because the destination stack block cannot be directly accessed along the stackside travel path.

Case 1: $\tau_{s s_{l}}(i, j)^{-1}=\frac{\left(W_{s}+W_{b s}\right)(i-j)+2 D_{s l}}{h_{v}}$

$$
\text { for } i \in N_{s}, j \in N_{s}, j \leq i
$$

Case 2: $\tau_{s s_{l}}(i, j)^{-1}=\frac{\left((i-1)\left(W_{s}+W_{b s}\right)+D_{e}+2 *\left(W_{l}-W_{b l}\right)+L_{l}+2 D_{e x}+D_{i n}\right.}{h_{v}}(33)$

$$
+\frac{\left.\delta\left(W_{s}+W_{b s}\right)+\left(W_{s}+W_{b} s\right) / 2+2 D_{s l}\right)}{h_{v}}
$$

$$
\begin{aligned}
\text { for } \delta=\left(N_{s}-N_{s r}\left(C_{s b}(i)\right)-j\right) ; i=1, \ldots, N_{s} ; j=1, \ldots, N_{s} ; j>i \\
\tau_{s s_{l}}^{-1}=\sum_{i=1}^{N_{s}} \sum_{j=1}^{N_{s}} \frac{1}{\left(N_{s}^{2}\right)} \tau_{s s_{l}}(i, j)^{-1}
\end{aligned}
$$

By using Equation 35 along with the expression for $\tau_{s q_{u}}^{-1}$ and the loading/unloading times, 
we can provide the expected service time expression for the $s l$ service type (Equation 36).

$$
\tau_{s l}^{-1}=\tau_{s s_{l}}^{-1}+\tau_{s q_{l}}^{-1}+L_{t}^{v}+U_{t}^{v}
$$

\section{B Estimating Stack Crane Service Times}

$$
\begin{aligned}
\mu_{s_{l}}^{-1}(i) & =\frac{p_{b}}{N_{b} N_{r}^{3}}\left(\sum _ { l _ { i } = 1 } ^ { l _ { i } = N _ { r } } \sum _ { l _ { k } = N _ { r } , m _ { k } = N _ { b } l _ { j } = m _ { r } = 1 } \sum _ { l _ { j } = 1 } \left(\max \left(\frac{\left|x_{l_{i}}-x_{l_{k}}\right|}{v_{s_{x}}}, \frac{\left|y_{1}-y_{m_{k}}\right|}{v_{s_{y}}}\right)\right.\right. \\
& \left.\left.+\max \left(\frac{\left|x_{l_{k}}-x_{l_{j}}\right|}{v_{s_{x}}}, \frac{\left|y_{m_{k}}-y_{1}\right|}{v_{s_{y}}}\right)\right)\right) \\
& +\frac{p_{i}}{N_{b}^{2} N_{r}^{3}}\left(\sum _ { l _ { i } = 1 , m _ { i } = 1 } ^ { l _ { i } = N _ { r } , m _ { i } = N _ { b } } \sum _ { l _ { k } = N _ { r } , m _ { k } = N _ { b } } ^ { l _ { j } = N _ { r } } \sum _ { l _ { k } = 1 , m _ { k } = 1 } \left(\max \left(\frac{\left|x_{l_{i}}-x_{l_{k}}\right|}{v_{s_{x}}}, \frac{\left|y_{m_{i}}-y_{m_{k}}\right|}{v_{s_{y}}}\right)\right.\right. \\
& \left.\left.+\max \left(\frac{\left|x_{l_{k}}-x_{l_{j}}\right|}{v_{s_{x}}}, \frac{\left|y_{m_{k}}-y_{1}\right|}{v_{s_{y}}}\right)\right)\right)+L_{t}^{s}+U_{t}^{s} \\
\mu_{s_{u}}^{-1}(i) & =\frac{p_{b}}{N_{b} N_{r}^{2}}\left(\sum_{l_{i}=1}^{l_{i}=N_{r} l_{j}=N_{r}, m_{j}=N_{b}}\left(\sum_{l_{j}=1, m_{j}=1}^{l_{j}}\left(\max \left(\frac{\left|x_{l_{i}}-x_{l_{j}}\right|}{v_{s_{x}}}, \frac{\left|y_{1}-y_{m_{j}}\right|}{v_{s_{y}}}\right)\right)\right)\right. \\
& +\frac{p_{i}}{N_{b}^{2} N_{r}^{3}}\left(\sum _ { l _ { i } = N _ { r } , m _ { i } = N _ { b } } ^ { l _ { l _ { k } = N _ { r } } l _ { j } = N _ { r } , m _ { j } = N _ { b } } \left(\max \left(\frac{\left|x_{l_{i}}-x_{l_{k}}\right|}{v_{s_{x}}}, \frac{\left|y_{m_{i}}-y_{1}\right|}{v_{s_{y}}}\right)\right.\right. \\
& \left.\left.+\max \left(\frac{\left|x_{l_{k}}-x_{l_{j}}\right|}{v_{s_{x}}}, \frac{\left|y_{1}-y_{m_{j}}\right|}{v_{s_{y}}}\right)\right)\right)+L_{t}^{s}+U_{t}^{s} \\
l_{l_{k}=1} & l_{j}=1, m_{j}=1
\end{aligned}
$$

From Equations 37 and 38, we can express the expected service time of the ASC resource (i), using Equation 39.

$$
\mu_{s}^{-1}(i)=p_{b} \mu_{s_{l}}^{-1}(i)+p_{i} \mu_{s_{u}}^{-1}(i)
$$




\section{Distribution of Absolute Percentage Errors}

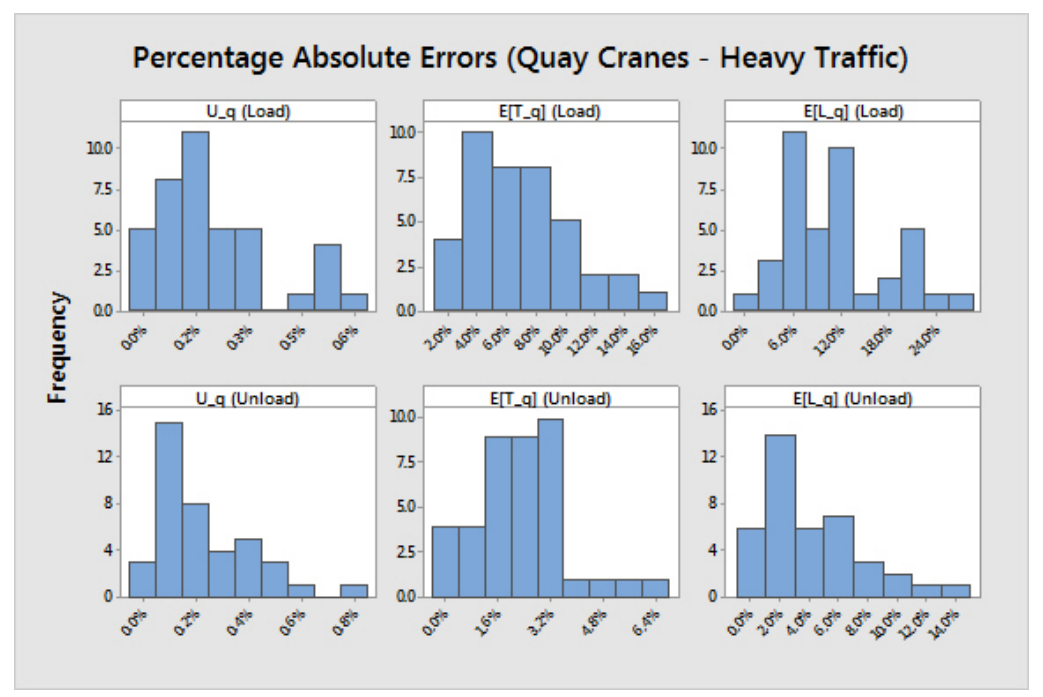

Figure C.1: Percentage absolute errors for the quay cranes with heavy traffic

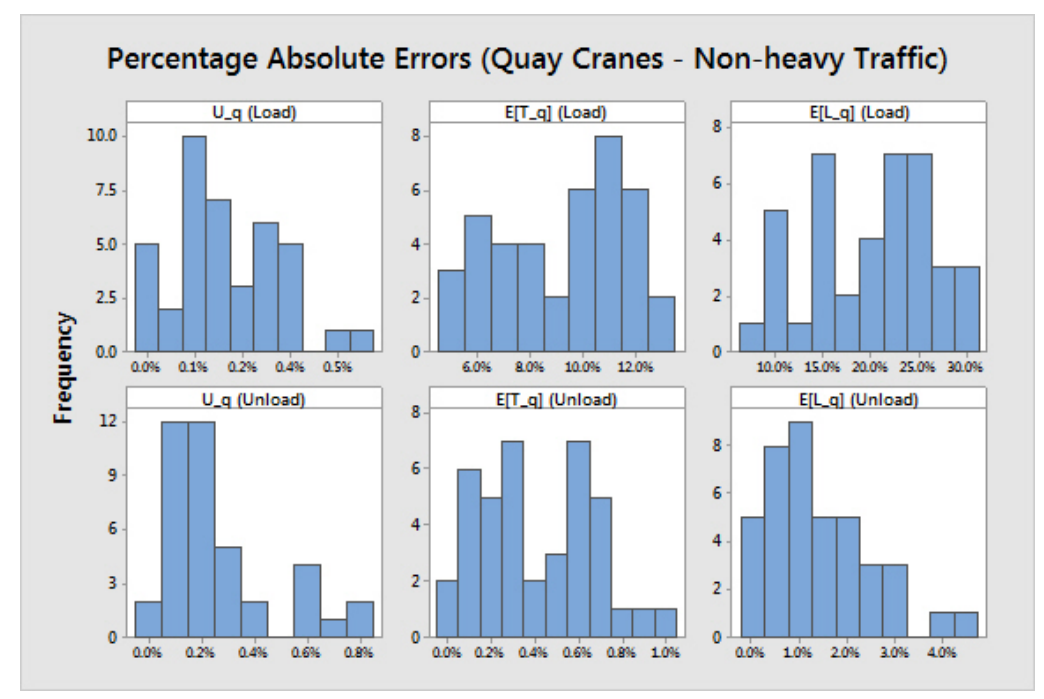

Figure C.2: Percentage absolute errors for the quay cranes with non-heavy traffic 


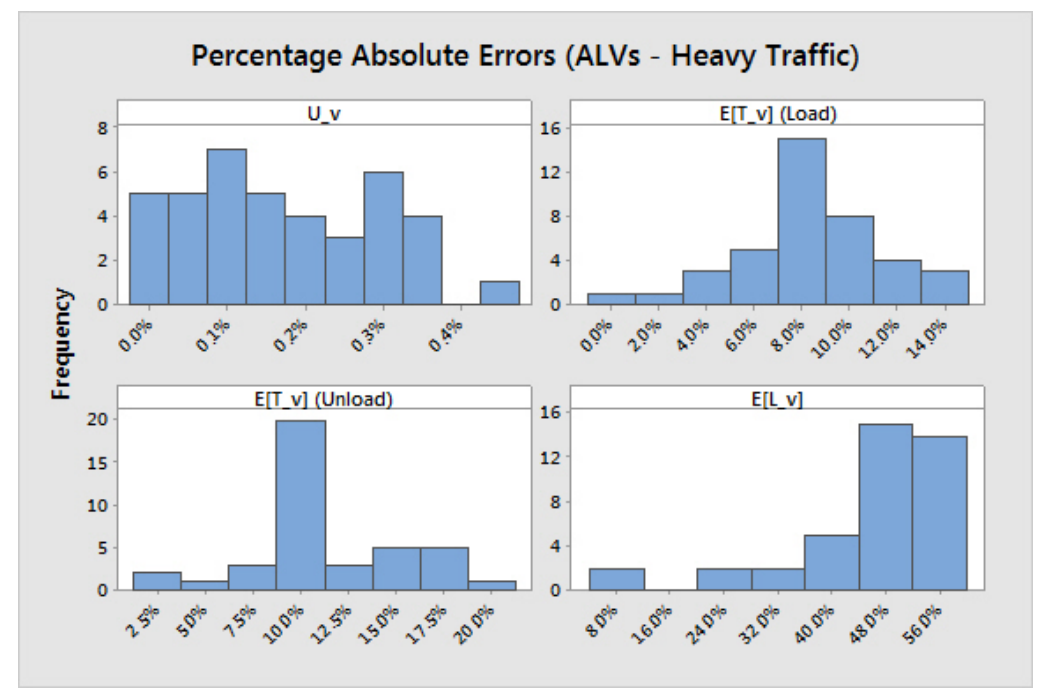

Figure C.3: Percentage absolute errors for the ALVs with heavy traffic

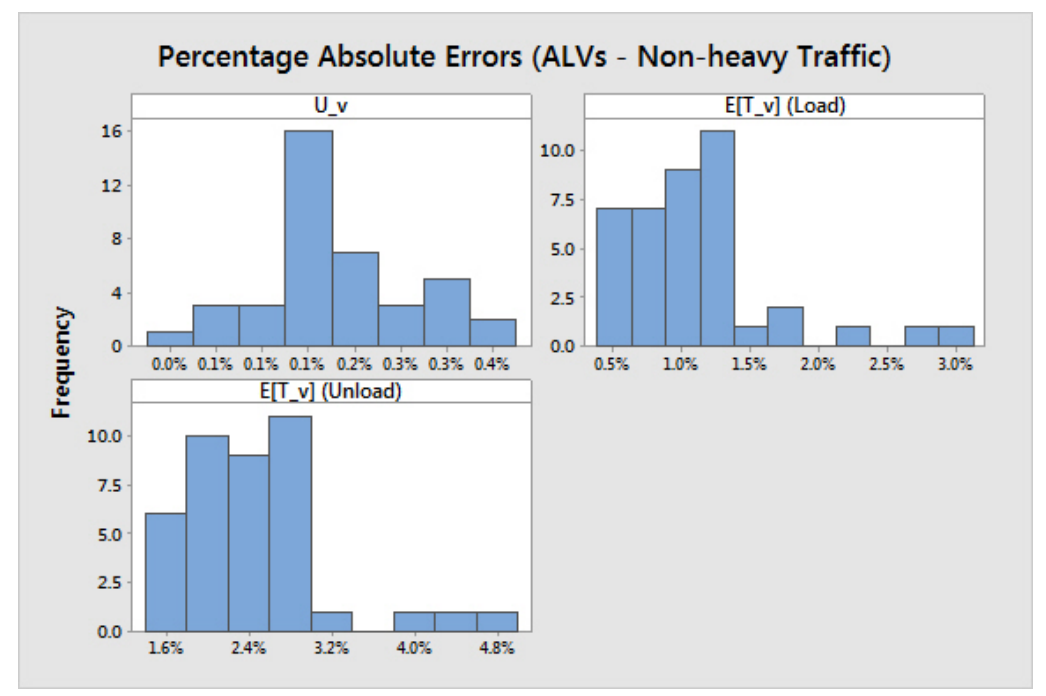

Figure C.4: Percentage absolute errors for the ALVs with non-heavy traffic 


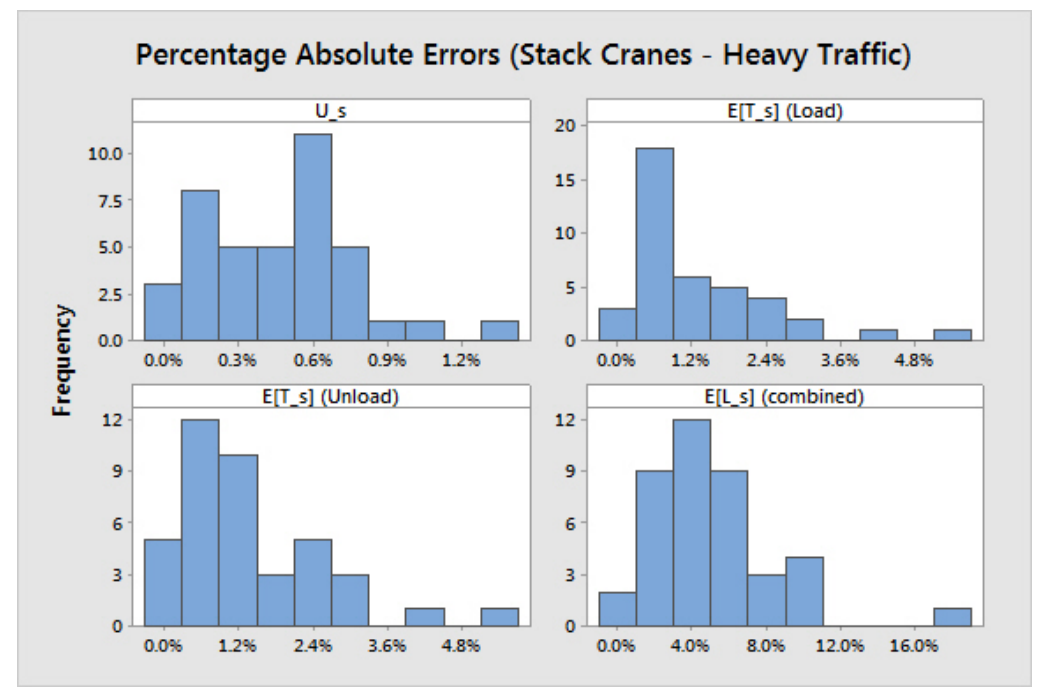

Figure C.5: Percentage absolute errors for the ASCs with heavy traffic

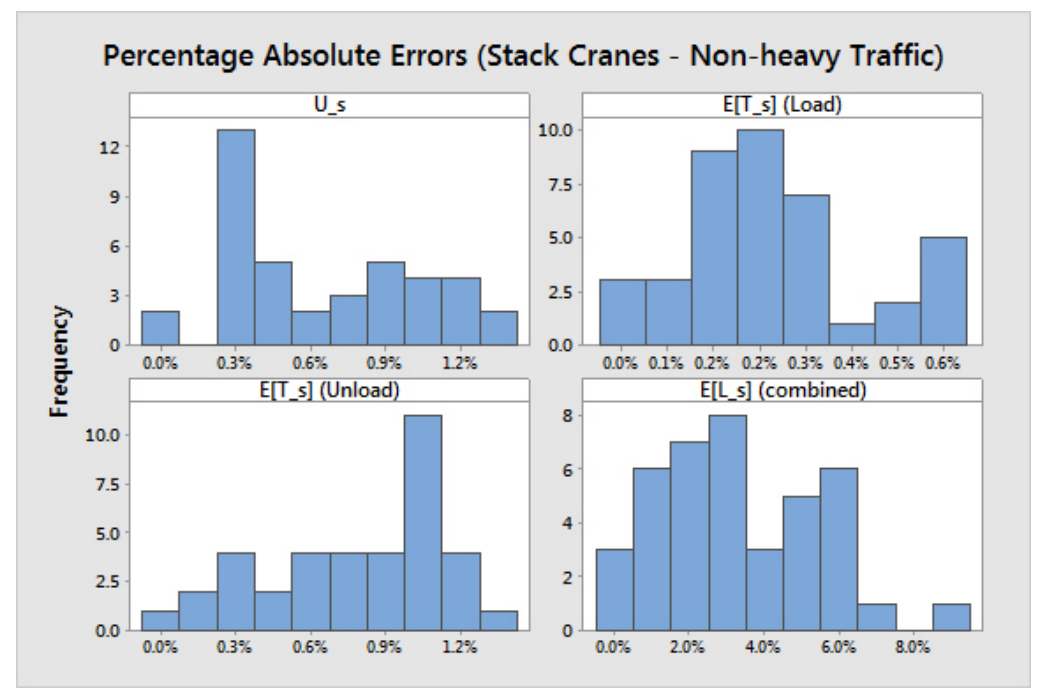

Figure C.6: Percentage absolute errors for the ASCs with non-heavy traffic 


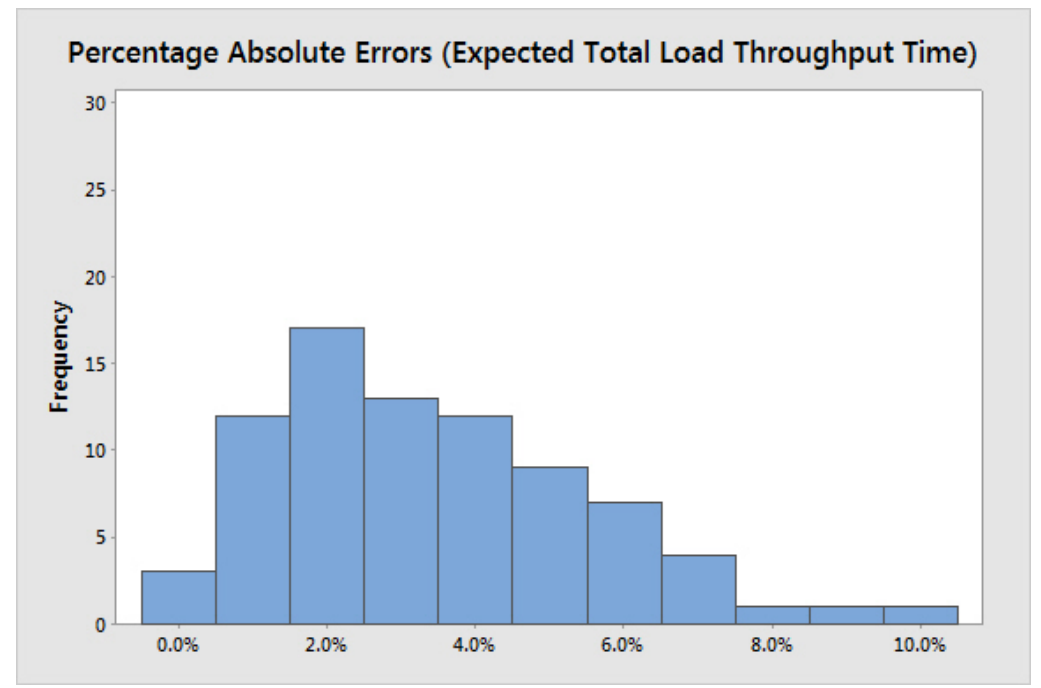

Figure C.7: Percentage absolute errors for the expected load throughput times

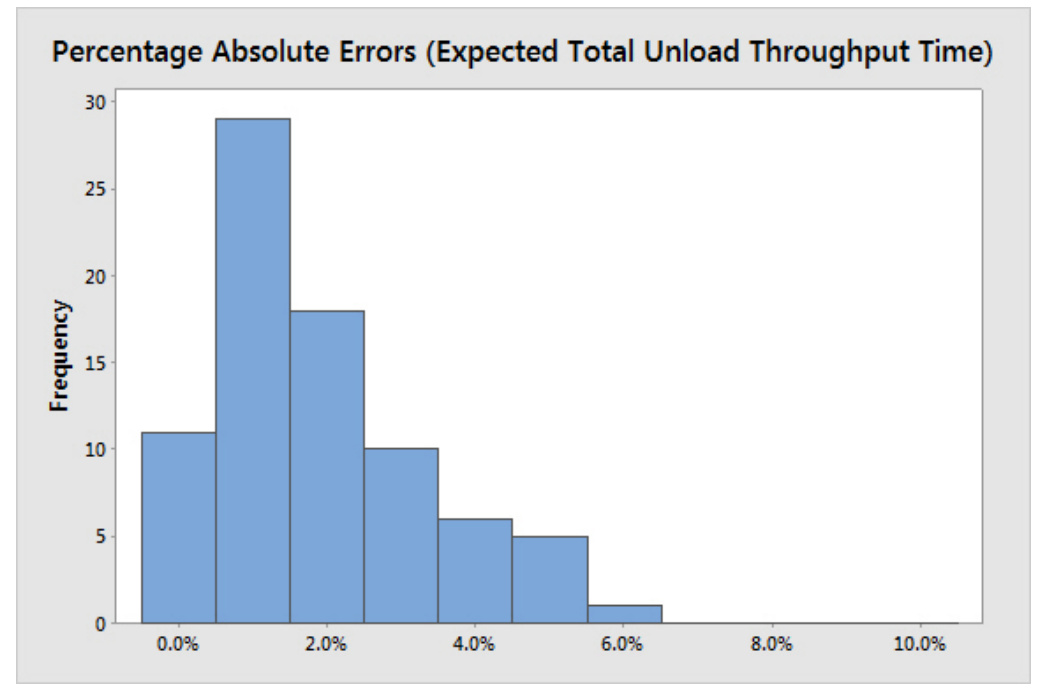

Figure C.8: Percentage absolute errors for the expected unload throughput times 


\begin{tabular}{|c|c|}
\hline \multicolumn{2}{|c|}{ ERIM Report Series Research in Management } \\
\hline ERIM Report Series reference number & ERS-2015-005-LIS \\
\hline Date of publication & $2015-04-10$ \\
\hline Version & $10-04-2015$ \\
\hline Number of pages & 48 \\
\hline Persistent URL for paper & http://hdl.handle.net/1765/78033 \\
\hline Email address corresponding author & debjit@iimahd.ernet.in \\
\hline Address & $\begin{array}{l}\text { Erasmus Research Institute of Management } \\
\text { (ERIM) } \\
\text { RSM Erasmus University / Erasmus School } \\
\text { of Economics } \\
\text { Erasmus University Rotterdam } \\
\text { PO Box } 1738 \\
3000 \text { DR Rotterdam, The Netherlands } \\
\text { Phone: +31104081182 } \\
\text { Fax: +31104089640 } \\
\text { Email: info@erim.eur.nl } \\
\text { Internet: http://www.erim.eur.nl }\end{array}$ \\
\hline Availability & 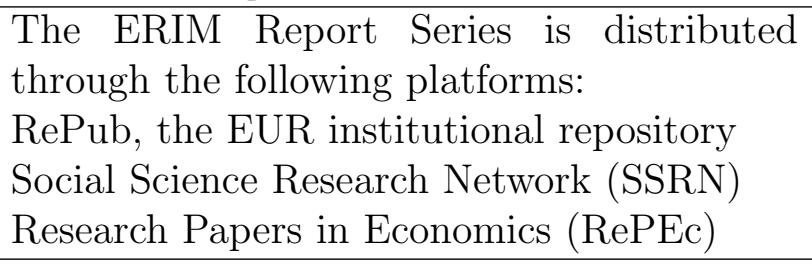 \\
\hline Classifications & $\begin{array}{l}\text { The electronic versions of the papers in the } \\
\text { ERIM Report Series contain bibliographic } \\
\text { metadata from the following classification } \\
\text { systems: } \\
\text { Library of Congress Classification (LCC) } \\
\text { Journal of Economic Literature (JEL) } \\
\text { ACM Computing Classification System } \\
\text { Inspec Classification Scheme (ICS) }\end{array}$ \\
\hline
\end{tabular}

